\title{
ATP13A2 deficiency disrupts lysosomal polyamine export
}

https://doi.org/10.1038/s41586-020-1968-7

Received: 21 December 2018

Accepted: 2 December 2019

Published online: 29 January 2020

\author{
Sarah van Veen ${ }^{1,4}$, Shaun Martin ${ }^{1,14}$, Chris Van den Haute ${ }^{2,3}$, Veronick Benoy', Joseph Lyons ${ }^{4}$, \\ Roeland Vanhoutte ${ }^{5}$, Jan Pascal Kahler ${ }^{5}$, Jean-Paul Decuypere ${ }^{1,6,8}$, , Géraldine Gelders $^{2}$, \\ Eric Lambie ${ }^{9,10}$, Jeffrey Zielich ${ }^{9}$, Johannes V. Swinnen ${ }^{6}$, Wim Annaert ${ }^{7}$, Patrizia Agostinis ${ }^{8,11}$, \\ Bart Ghesquière $^{12}$, Steven Verhelst ${ }^{5,13}$, Veerle Baekelandt ${ }^{2}$, Jan Eggermont ${ }^{1}$ \& \\ Peter Vangheluwe ${ }^{1 *}$
}

\begin{abstract}
ATP13A2 (PARK9) is a late endolysosomal transporter that is genetically implicated in a spectrum of neurodegenerative disorders, including Kufor-Rakeb syndrome-a parkinsonism with dementia ${ }^{1}$-and early-onset Parkinson's disease ${ }^{2}$. ATP13A2 offers protection against genetic and environmental risk factors of Parkinson's disease, whereas loss of ATP13A2 compromises lysosomes ${ }^{3}$. However, the transport function of ATP13A2 in lysosomes remains unclear. Here we establish ATP13A2 as a lysosomal polyamine exporter that shows the highest affinity for spermine among the polyamines examined. Polyamines stimulate the activity of purified ATP13A2, whereas ATP13A2 mutants that are implicated in disease are functionally impaired to a degree that correlates with the disease phenotype. ATP13A2 promotes the cellular uptake of polyamines by endocytosis and transports them into the cytosol, highlighting a role for endolysosomes in the uptake of polyamines into cells. At high concentrations polyamines induce cell toxicity, which is exacerbated by ATP13A2 loss due to lysosomal dysfunction, lysosomal rupture and cathepsin B activation. This phenotype is recapitulated in neurons and nematodes with impaired expression of ATP13A2 or its orthologues. We present defective lysosomal polyamine export as a mechanism for lysosome-dependent cell death that may be implicated in neurodegeneration, and shed light on the molecular identity of the mammalian polyamine transport system.
\end{abstract}

ATP13A2 is a P5B-ATPase that belongs to the family of P-type ATPases, which couple ATP hydrolysis to substrate transport while transiently forming a catalytic phospho-intermediate ${ }^{4}$. ATP13A2 is generally described as a heavy-metal transporter ${ }^{5}$, but $\mathrm{Ca}^{2+}$ (ref. ${ }^{6}$ ) and the polyamine spermidine (SPD) $)^{7,8}$ have also been proposed as potential substrates. To screen for the transported substrate(s) of ATP13A2, we measured ATPase activity in the presence of various candidate substrates in solubilized microsomal membrane fractions of SH-SY5Y cells that overexpress wild-type human ATP13A2 (hereafter denoted WT-OE) or comparable levels of the catalytically dead mutant ATP13A2(D508N) (containing an aspartic-acid-to-asparagine mutation at position 508; denoted D508N-OE) $)^{9,10}$.

The ATPase activity of wild-type ATP13A2 was significantly stimulated by the polyamines SPD and spermine (SPM) (Fig. 1a), whereas SPM had no effect on the activity of the D508N mutant (Extended Data Fig. 1a). $\mathrm{MnCl}_{2}, \mathrm{ZnCl}_{2}, \mathrm{FeCl}_{3}, \mathrm{CaCl}_{2}$, diamines, monoamines and amino acids exerted no effect on activity (Extended Data Fig. 1a-d), but the polyamines SPM, $N^{1}$-acetylspermine and SPD stimulated the ATPase activity of ATP13A2 in a concentration-dependent manner (Fig. 1b, Extended Data Fig. 1e), with the highest apparent affinity observed for SPM (Extended Data Table 1).

The catalytic autophosphorylation and/or dephosphorylation reactions of P-type ATPases occur in response to binding of the transported substrate ${ }^{4}$. ATP13A2 forms a phospho-intermediate on the D508 residue in the absence of SPM supplementation ${ }^{9,10}$, whereas the addition of SPM leads to a dose-dependent reduction in the levels of the ATP13A2 phosphoenzyme (Fig. 1c), which is not seen with ornithine (Extended Data Fig. 1f). The dephosphorylation rate after a chase with non-radioactive ATP increased in the presence of SPM (Fig. 1d), which further indicates that SPM could be the transported substrate.

SPM also stimulated the ATPase activity of purified human ATP13A2 (for purification, see Extended Data Fig. 2a-e). However, purified

'Laboratory of Cellular Transport Systems, Department of Cellular and Molecular Medicine, KU Leuven, Leuven, Belgium. ${ }^{2}$ Laboratory for Neurobiology and Gene Therapy, Department of Neurosciences, KU Leuven, Leuven, Belgium. ${ }^{3}$ Leuven Viral Vector Core, KU Leuven, Leuven, Belgium. ${ }^{4}$ Department of Molecular Biology and Genetics - DANDRITE, Aarhus, Denmark. ${ }^{5}$ Laboratory of Chemical Biology, Department of Cellular and Molecular Medicine, KU Leuven, Leuven, Belgium. ${ }^{6}$ Laboratory of Lipid Metabolism and Cancer, Department of Oncology, LKI-



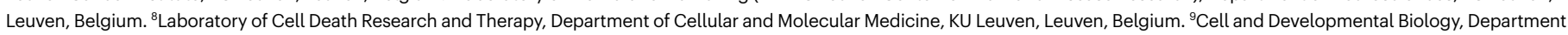
Biology II, Ludwig-Maximilians-Universität Munich, Munich, Germany. ${ }^{10}$ Department of Cell and Developmental Biology, University College London, London, UK. "VIB-KU Leuven Center for Cancer Biology, Department of Oncology, KU Leuven, Leuven, Belgium. ${ }^{2}$ Metabolomics Expertise Center (VIB-KU Leuven Center for Cancer Biology), Department of Oncology, KU Leuven, Leuven, Belgium. ${ }^{13}$ Leibniz Institute for Analytical Sciences ISAS, Dortmund, Germany. ${ }^{14}$ These authors contributed equally: Sarah van Veen, Shaun Martin. *e-mail: peter.vangheluwe@ kuleuven.be 




$\mathbf{f}$

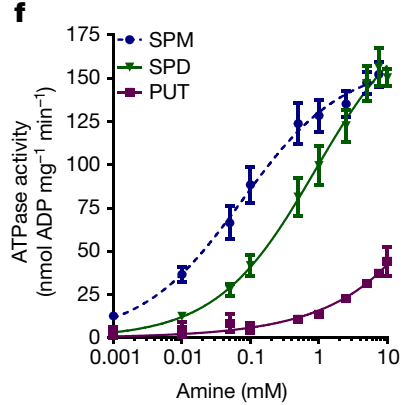<smiles>NCCCCNCCCCN</smiles>

PUT

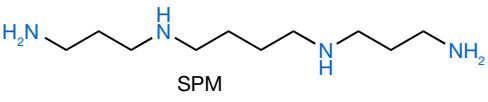

SPM c

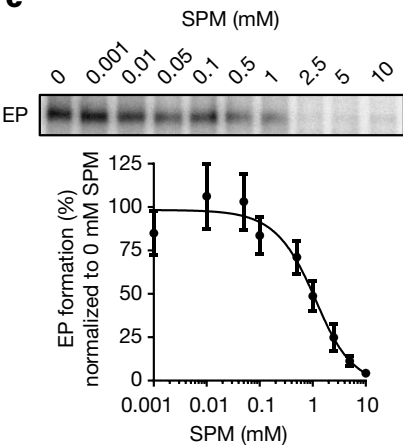

g $\mathbf{h}$

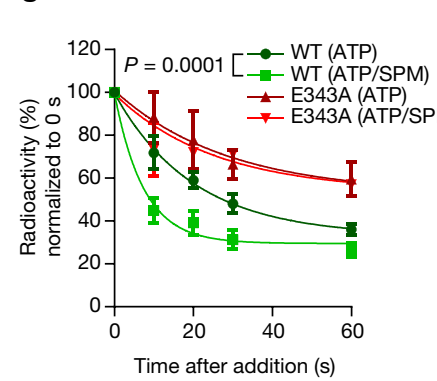

Fig. 1 ATP13A2 is a polyamine transporter. a, Chemical structures of ornithine (ORN), putrescine (PUT), spermidine (SPD) and spermine (SPM). b-d, Measurements on solubilized SH-SY5Y microsomes overexpressing ATP13A2 (WT-OE).b, Dose-response curves showing the effect of SPM, SPD, PUT and ORN on the ATPase activity of ATP13A2. c, ATP13A2 phosphoenzyme (EP) levels in WT-OE cells in the presence of increasing SPM concentrations. Top, representative autoradiogram; bottom, quantification of EP. d, Pulse ([ $\left.\left.\gamma^{-32} \mathrm{P}\right] \mathrm{ATP}\right)$ chase (cold ATP) of dephosphorylation in the presence and absence of $1 \mathrm{mMSPM}$ in WT-OE microsomes. e, ATPase activity of purified ATP13A2 under increasing concentrations of SPM (in the presence and absence of phosphatidic acid (PA) and/or PtdIns $(3,5) \mathrm{P}_{2}$ ). f, Dose-response curves showing the effect of SPM, SPD and PUT on the ATPase activity of purified ATP13A2 supplemented with phosphatidic acid and $P \operatorname{td} I n s(3,5) \mathrm{P}_{2}$ (as a reference, $\mathrm{SPM}+$ phosphatidic acid + PtdIns $(3,5) \mathrm{P}_{2}$ from e is also shown). g, Pulse ([ $\left.\gamma^{-32} \mathrm{P}\right]$ ATP) chase (cold ATP) of dephosphorylation measured for

ATP13A2 presented similar properties to microsomal ATP13A2 only in the presence of the regulatory lipids phosphatidylinositol $(3,5)$ bisphosphate $\left(\operatorname{Ptd} I n s(3,5) \mathrm{P}_{2}\right.$ ) and phosphatidic acid, which bind to the $\mathrm{N}$ terminus of ATP13A2 ${ }^{9-11}$ (Fig. 1e, f, Extended Data Fig. 2f, Extended Data Table 1).SPM-induced ATPase activity was blocked by orthovanadate, a general P-type ATPase inhibitor (Extended Data Fig.1g). Finally, we also purified the mutant ATP13A2(E343A), which carries a mutation in the conserved catalytic motif for dephosphorylation $\left({ }^{341} \mathrm{TGES}\right)$ (Extended Data Fig. 2g). The E343A mutant underwent autophosphorylation (Fig. 1g) but displayed limited SPM-induced ATPase activity (Extended Data Fig. 2h). Notably, when the phosphoenzyme was chased with cold ATP, SPM clearly stimulated dephosphorylation of purified wild-type ATP13A2, but not of the E343A mutant (Fig.1g, Extended Data Fig.1h, 6a).

\section{ATP13A2 is a lysosomal polyamine exporter}

Next, we performed transport assays with ${ }^{3} \mathrm{H}$-labelled SPM $\left({ }^{3} \mathrm{H}-\mathrm{SPM}\right)$ in reconstituted vesicles from solubilized yeast membranes expressing biotin acceptor domain (BAD)-labelled wild-type ATP13A2 or the

d

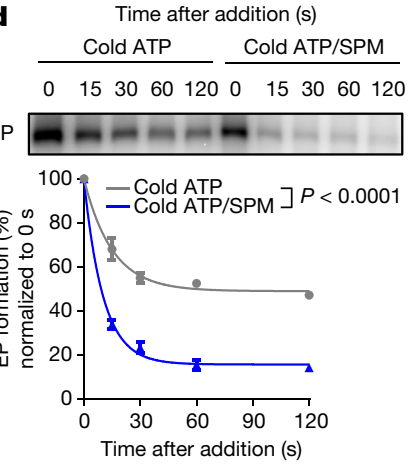

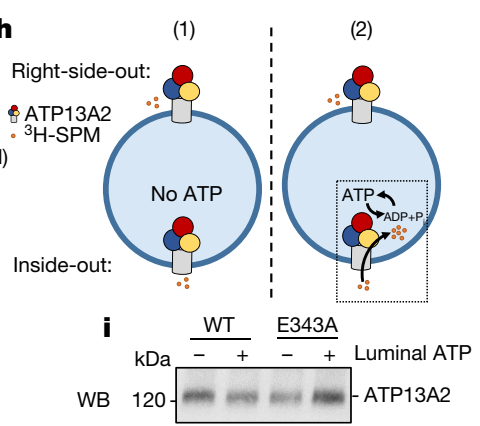

e


purified ATP13A2 (wild-type or E343A mutant) in the presence or absence of 1 mMSPM. h, Illustration of vesicle reconstitution and the ${ }^{3} \mathrm{H}$-spermine $\left({ }^{3} \mathrm{H}\right.$-SPM) transport assay. $\mathbf{i}$, Immunoblot of reconstituted vesicles.j, Uptake of ${ }^{3} \mathrm{H}$-SPM in reconstituted vesicles derived from yeast overexpressing BAD-tagged ATP13A2 (wild-type or E343A mutant) supplemented with phosphatidylcholine and phosphatidic acid, in the presence or absence of intraluminal ATP and an ATP-regenerating system. Data are presented as mean \pm s.e.m. in b-g, or as box and whisker plots with overlaid individual data points representing replicates (j; horizontal line, median; box boundaries, 25 th and 75 th percentiles). The number of independent biological experiments were as follows: $n=3 \mathbf{b}$ (ORN, SPD), d-g (E343A), i,j (wild-type ATP outside;E343A); $n=4 \mathbf{c}, \mathbf{e}$ (no lipid); $n=5 \mathbf{b}$ (PUT), $\mathbf{g}$ (wild-type); $n=6 \mathbf{j}$ (wild-type no ATP), $\mathbf{b}$ (SPM); $n=8 \mathbf{j}$ (wild-type ATP inside). Analysis by two-way (g) or one-way (j) ANOVA with Tukey's corrections. Fitted lines indicate nonlinear allosteric sigmoidal (b,e,f) or twophase (c, d, g) decay. For gel source data, see Supplementary Fig. 1.

mutant ATP13A2(E343A), supplemented with the activating lipid phosphatidic acid. This reconstitution rendered two populations of ATP13A2 proteins that were inserted either right-side-out (ATP-binding domain in the extraluminal space) or inside-out (ATP-binding domain in the lumen) (Fig. 1h, i). Uptake of ${ }^{3} \mathrm{H}$-SPM was detected for wild-type ATP13A2-but not for the E343A mutant-only when ATP was present inside the vesicles, together with an ATP-regenerating system (Fig. 1j); that is, when ATP13A2 was positioned inside-out ((2) in Fig. 1h). Extending these insights to the cellular context, in which ATP13A2 is present in the late endolysosomal compartment ${ }^{1,12}$, ATP13A2 most probably operates as a lysosomal SPM exporter.

Notably, the functionality of ATP13A2 affects the cellular polyamine content. We generated two independent ATP13A2-knockout SH-SY5Y cell lines using CRISPR-Cas9 genome editing (denoted KO; Extended Data Fig. 3a) and, upon analysis by mass spectrometry, demonstrated that the total cellular polyamine content was lower in KO than in control cells (Fig. 2a). Expression of either the wild-type ATP13A2 in the $\mathrm{KO}$ background (denoted KO/WT) for rescue, or the D508N mutant $(\mathrm{KO} / \mathrm{D} 508 \mathrm{~N})$ as a negative control (Extended Data Fig. 3b), resulted in 

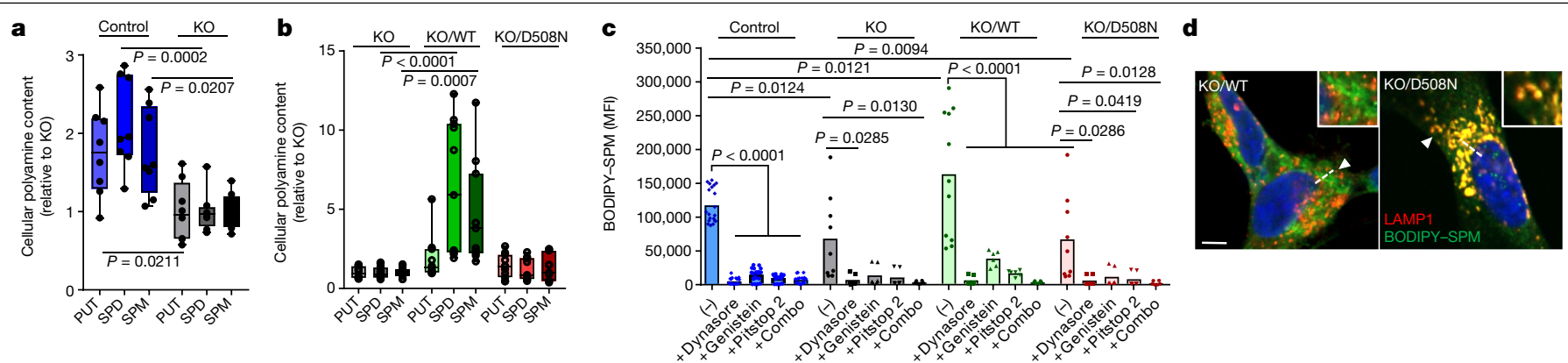

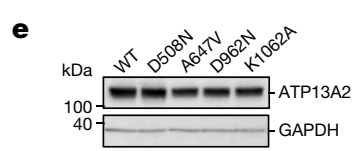

$\mathbf{f}$
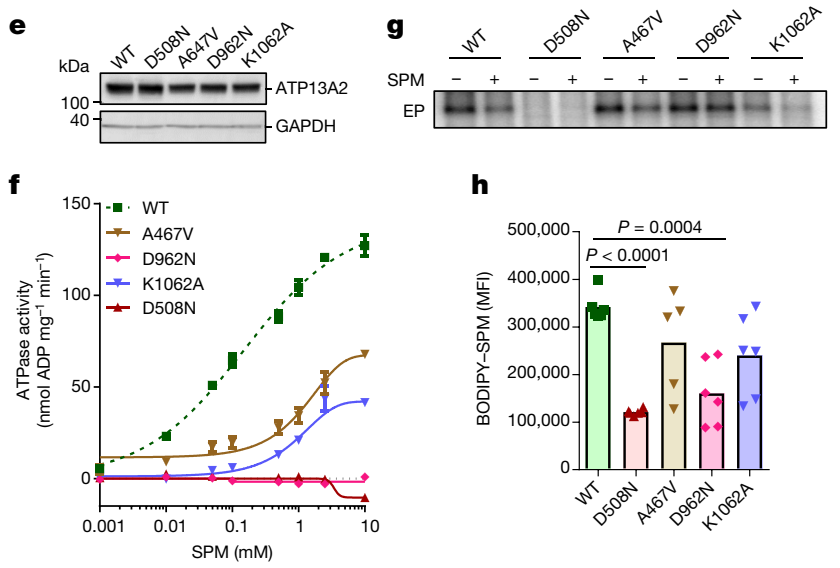

h

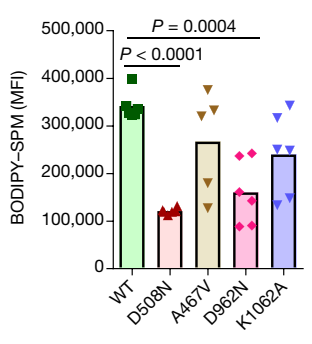

Fig. 2 |ATP13A2 transport affects cellular polyamine uptake, which is impaired by catalytic and disease mutations. a, b, Metabolomics of cellular polyamines in ATP13A2 knockout cells (KO) compared with SH-SY5Y controls (a), or compared with rescue cell lines expressing wild-type ATP13A2 (KO/WT) or ATP13A2(D508N) (KO/D508N) (b). c, Uptake of BODIPY-SPM in the presence or absence of the endocytosis inhibitors Dynasore, genistein and/or Pitstop 2, alone or in combination (combo). d, Confocal microscopy of BODIPY-SPM distribution (see Methods). Scale bar, $5 \mu \mathrm{m}$. The arrow head shows the region that is expanded in the insets; the dashed line shows the region analysed in Extended Data Fig. 1j.e,f, Immunoblotting (e) and SPM-induced ATPase activity (f) of microsomes expressing wild-type ATP13A2, the D508N mutant, or catalytic mutants in M4 (A467V), M6 (D962N) and M8 (K1062A) ${ }^{30}$. g, Representative autoradiogram depicts phosphoenzymes of wild-type ATP13A2 compared with the indicated mutants in the presence or absence of $1 \mathrm{mM}$ SPM. h, Uptake of BODIPY-SPM in cells expressing the indicated mutant proteins. i,j, Immunoblotting (i) or SPM-induced ATPase activity (j) of SH-SY5Y microsomes overexpressing mutants associated with Kufor-Rakeb syndrome (T512I and G872R) or early-onset Parkinson's disease (T12M, G528R, A741T). i

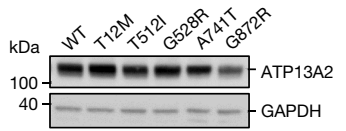

j

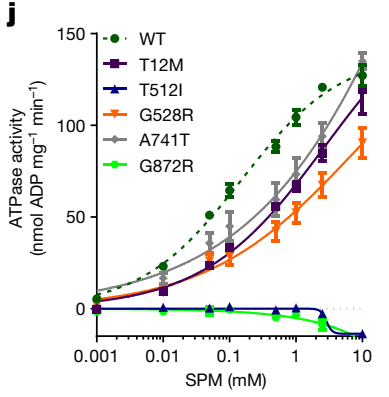



】

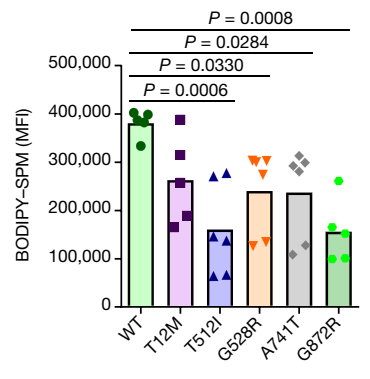

The SPM dose-response curve from Fig. $1 b$ is shown as a reference in $\mathbf{f}$ and $\mathbf{j}$. $\mathbf{k}$, Representative autoradiogram depicting phosphoenzymes of wild-type ATP13A2 compared with the disease-related mutants in the presence and absence of 1 mM SPM. I, BODIPY-SPM uptake in cells containing the indicated mutant proteins. MFI, mean fluorescence intensities; $(-)$, vehicle-treated sample. Data are presented as individual data points (representing replicates) overlaid on box and whisker plots (a, b, horizontal line, median; box boundaries, 25 th and 75 th percentiles) or mean (c, h, $\mathbf{l})$ or mean \pm s.e.m. $(\mathbf{f}, \mathbf{j})$. The number of independent biological experiments were as follows: $n=3 \mathbf{d}-\mathbf{f}$, g (D508N, A467V, D962N, K1062A (SPM)), h,j (T12M, T512I, G872R), k (T12M (-), T12M, T512I, G528R, A741T, G872R (SPM)), l; $n=4$ a-c(KO, KO/WT, KO/DN), g(D508N, A467V, D962N, K1062A (-)), i,j (A741T, G528R), k (wild-type, T512I, G528R, A741T, G872R (-)); $n=5 \mathbf{c}$ (control), g (wild-type (-)); $n=6 \mathbf{g}$ (wild-type (SPM)), k (wild-type (SPM)). Analysis was performed using one-way ANOVA with Tukey's (a-c) or Dunnett's (h-l) corrections. Fitted lines indicate nonlinear allosteric sigmoidal (f(D962N, D508N),j) or one-phase (f(A467V, K1062A)) association. For gel source data see Supplementary Fig. 1.

a significantly higher SPD and SPM content in KO/WT cells than in KO or KO/D508N cells (Fig. 2b).

Flow cytometry experiments revealed that ATP13A2 promotes the cellular uptake of BODIPY-labelled polyamines ${ }^{13}$. WT-OE cells took up more BODIPY-SPD and BODIPY-SPM than D508N-OE cells or control cells that expressed firefly luciferase (Fluc; Extended Data Fig. 4a, b). Similarly, KO/WT cells displayed a twofold higher uptake of BODIPYSPM than ATP13A2 KO and KO/D508N cells, both of which took up less than control cells (Fig. 2c). The comparable uptake of the fluorescein isothiocyanate (FITC)-dextran conjugate in the KO/WT and the KO/ D508N cells (Extended Data Fig. 4c) demonstrated that the higher BODIPY-SPM uptake in KO/WT cells compared with KO/D508N cells is not explained by an increased endocytic rate, but depends on the transport activity of ATP13A2. The stimulatory effect of ATP13A2 on endocytosis appears as a transport-independent phenotype ${ }^{14}$.

The observation that ATP13A2 transports SPM towards the cytosol (Fig. 1j) and increases the cellular SPM content (Fig. 2a, b) suggests that it could transport endocytosed polyamines into the cytosol. Indeed, endocytosis inhibitors prevented the uptake of FITC-dextran
(Extended Data Fig. 4c) and blocked that of BODIPY-SPM (Fig. 2c). Using confocal microscopy, we confirmed that KO/WT cells had a higher BODIPY-SPM content than KO/D508N cells (Extended Data Fig. 1i). In the KO/D508N cells, BODIPY-SPM mainly colocalized with LAMP1positive vesicles (Fig. 2d, Extended Data Fig. 1j, k); this is indicative of accumulation in the late endolysosomes, which is not a lysosomotropic effect ${ }^{15}$. By contrast, cells with functional ATP13A2 displayed a broader distribution of BODIPY-SPM, and it was more abundant in the cytosol and nucleus; this is consistent with the transport direction from lysosomal lumen to cytosol (Fig. 2d, Extended Data Fig. 11). By stimulating cellular SPM uptake and transporting SPM into the cytosol, ATP13A2 complements endogenous SPM synthesis, which depends on the enzymes ornithine decarboxylase (ODC) and the SPD and SPM synthases. Consistent with this, KO/WT cells were protected against pharmacological inhibition of ODC and the SPD and SPM synthases, and a lack of ATP13A2 activity sensitized $\mathrm{KO}$ and $\mathrm{KO} / \mathrm{D} 508 \mathrm{~N}$ cells to inhibition (Extended Data Fig. $4 \mathrm{~d}-\mathrm{g}$ ); this is consistent with the negative genetic interactions between ATP13A2 orthologues and ODC in yeast ${ }^{16}$ and Caenorhabditis elegans ${ }^{8}$. 

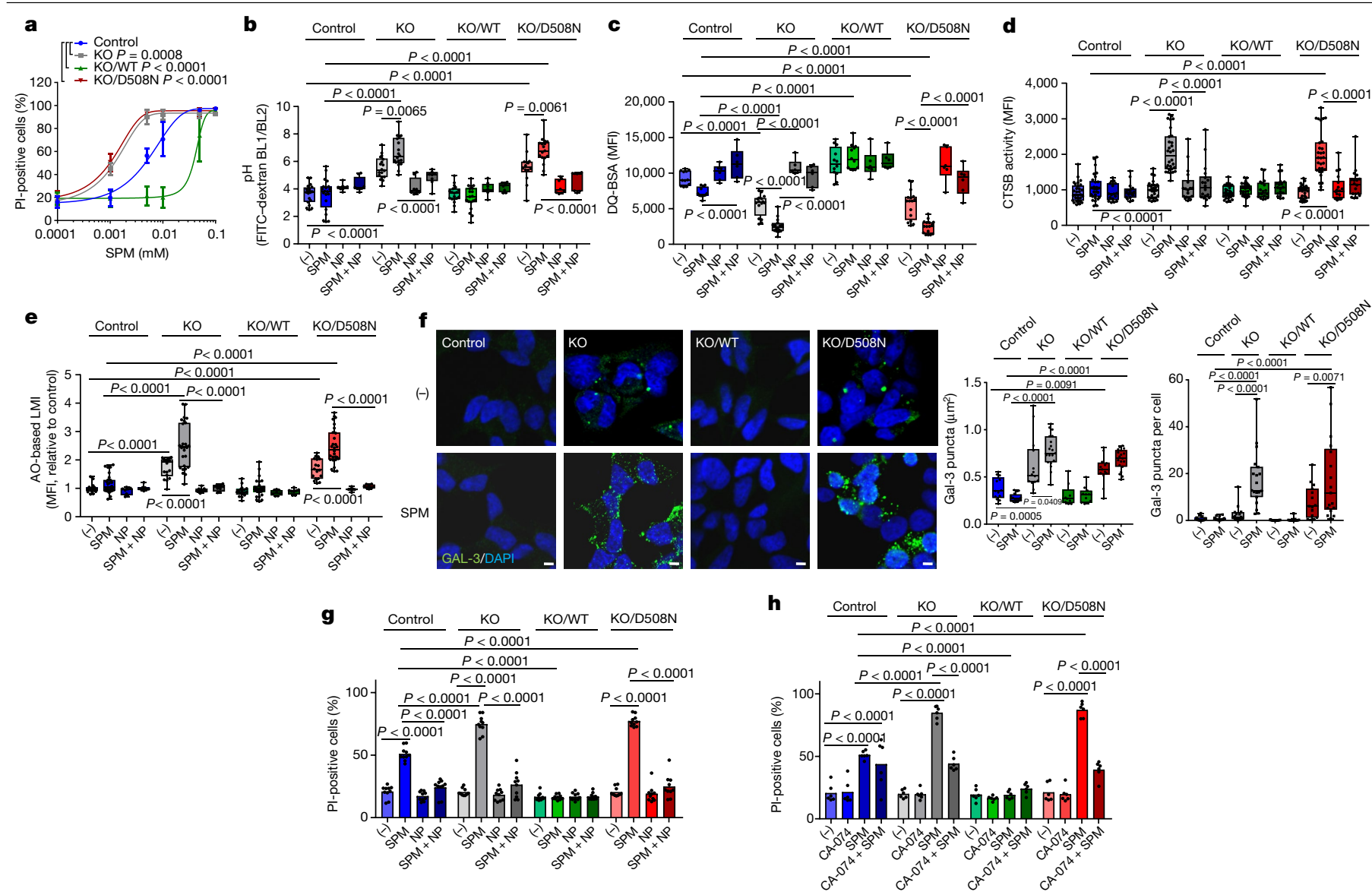

Fig. 3 | ATP13A2 protects against lysosome-dependent SPM toxicity. a, SPM-induced cell death (PI, propidium iodide) in SH-SY5Y control, ATP13A2 knockout (KO) and rescue cell lines with wild-type ATP13A2 (KO/WT) or ATP13A2(D508N) (KO/D508N). b-f, Effect of SPM $(10 \mu \mathrm{M}, 4 \mathrm{~h})$ and acidic nanoparticles (NP, 100-nm diameter; Extended Data Fig. 8j) on lysosomal functionality. b, Lysosomal $\mathrm{pH}$ measured by ratiometric FITC-dextran (standard curve, Extended Data Fig. 8i).c, d, Lysosomal degradation capacity analysed by DQ-BSA (c) or cathepsin B (CTSB) (d) activity. e, f, Assessment of lysosomal membrane integrity (LMI) via acridine orange (AO) staining (e), or galectin-3 (Gal-3) punctae formation (lysosomal rupture) (f). In $\mathbf{f}$, the confocal images depict representative images (DAPI staining for nuclei reference; scale

\section{Mutations disrupt SPM-induced ATP13A2 activity}

We next used mutagenesis techniques to confirm that the SPM-dependent activation of ATP13A2 depends on residues in the predicted substratebinding site near transmembrane segment M4. We introduced mutations in transmembrane segments M4 (A467V), M6 (D962N) and M8 (K1062A) (Fig. 2e, Extended Data Fig. 5a, b; see Methods for the rationale in choosing these residues). In comparison with the wild type, the three mutants displayed a lower SPM-induced ATPase activity and apparent affinity for SPM (Fig. 2f), as well as a reduced cellular uptake of BODIPY-SPD and BODIPY-SPM (Extended Data Fig. 5c, Fig. 2h), which suggests that these residues contribute to SPM coordination in the membrane region. SPMinduced dephosphorylation was completely abolished in the D962N mutant (Fig. 2g, Extended Data Figs. 5d, 6), indicating that D962 may couple SPM binding to the dephosphorylation reaction.

More than thirty disease-associated mutations have been identified in $A T P 13 A 2$ (Extended Data Fig. 7). We determined the activity of mainly lysosomal-localized mutants of ATP13A2 arising from point mutations in ATP13A2 that are linked to early-onset Parkinson's disease or KuforRakeb syndrome. The T512I and G872R mutants-associated with KuforRakeb syndrome-did not exhibit ATPase or autophosphorylation bars, $2.5 \mu \mathrm{m})$. The box and whisker plots depict the size (left) and number (right) of punctae.g, h, The effect of nanoparticles (g) or the cathepsin B inhibitor CA-074 $(25 \mu \mathrm{M})(\mathbf{h})$ on SPM-induced cytotoxicity. Data are presented as individual data points (representing replicates) overlaid on box and whisker plots (b-f, horizontal line, median; box boundaries, 25 th and 75 th percentiles) or means (g, h) or mean \pm s.e.m. (a). The number of individual biological experiments were as follows: $n=3 \mathbf{a}, \mathbf{b}-\mathbf{d}(\mathrm{NP}, \mathrm{NP}+\mathrm{SPM}), \mathbf{f}-\mathbf{h} ; \boldsymbol{n}=4 \mathbf{e}$, $(\mathrm{NP}, \mathrm{NP}+\mathrm{SPM}) ; n=6 \mathbf{b}-\mathbf{d}((-), \mathrm{SPM}) ; n=7 \mathbf{e}((-), \mathrm{SPM})$. Analysis was performed using two-way ANOVA with Dunnett's test (a) or one-way ANOVA with Tukey's (b-e, g, h) or Sidak's (f) test. Fitted lines indicate nonlinear log(inhibitor) versus response (variable slope) (a). activity and were SPM-insensitive, which is in line with their strongly reduced BODIPY-SPD and BODIPY-SPM uptake (Fig. 2i-1, Extended Data Fig. 5e-g). The T12M, G528R and A741T mutants-associated with early-onset Parkinson's disease-had a less severe effect. Compared with wild type, a reduction in the apparent SPM affinity was observed and phosphoenzyme levels were more (T12M) or less (G528R and A741T) sensitive to SPM. In cells, the uptake of BODIPY-SPD and BODIPY-SPM was less severely impaired with the mutations associated with earlyonset Parkinson's disease than those associated with Kufor-Rakeb syndrome (Fig. 2i-l, Extended Data Fig. 5e-g). In summary, ATP13A2dependent polyamine transport is disturbed in all mutants tested, and the degree of functional impact correlates with the phenotypic differences between early-onset Parkinson's disease and Kufor-Rakeb syndrome; however, the mutation type is not the sole determinant of the clinical phenotype ${ }^{17}$.

\section{ATP13A2 protects against polyamine toxicity}

We further investigated whether defective lysosomal polyamine export explains the lysosomal phenotype in ATP13A2 KO cells ${ }^{3}$. SPM and SPD are abundant organic polycations that support cell function, but at 

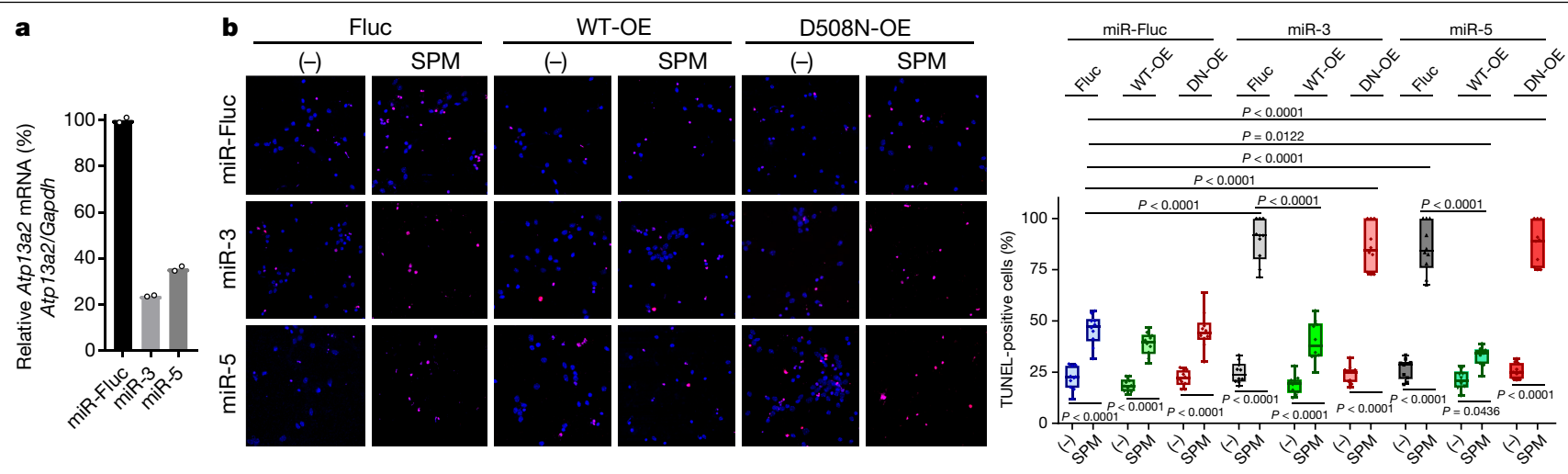

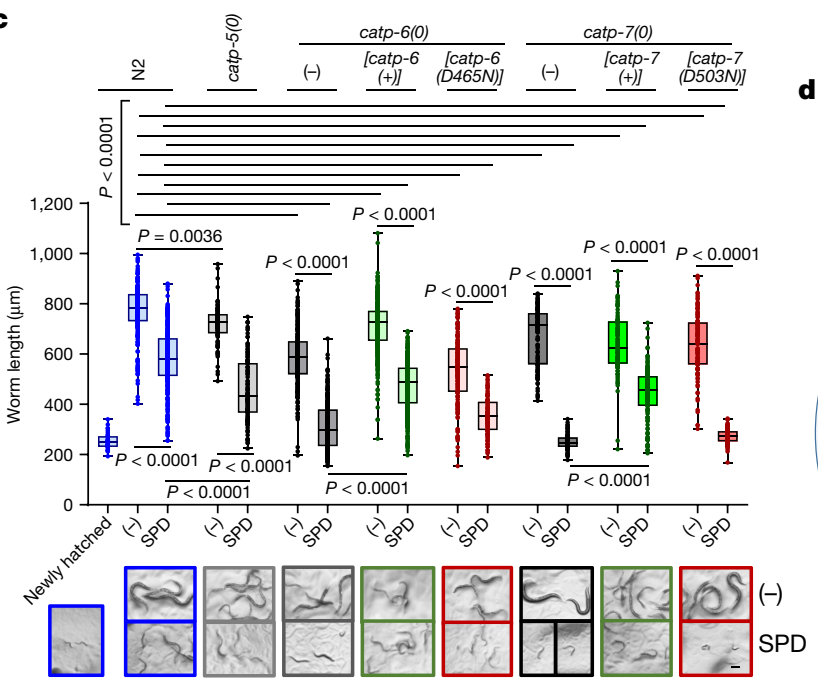

Fig. 4 | Loss of ATP13A2 orthologues exacerbates the toxicity of polyamines in primary neurons and in C.elegans. a, Lentiviral knockdown of Atp13a2 in isolated mouse cortical neurons (miR-3 or miR-5) was confirmed by quantitative PCR relative to Gapdh. miRNA against firefly luciferase (miR-Fluc) was used as a negative control.b, miR-Fluc and Atp13a2 knockdown neurons were transduced with Fluc, wild-type human ATP13A2 or ATP13A2(D508N). SPM-induced cytotoxicity was assayed via TUNEL staining. Left, representative confocal images depicting TUNEL-positive cells; right, box and whisker plots showing quantification of the TUNEL staining. c, The indicated worm strains were assessed for SPD toxicity. Worm lengths were determined as a read-out

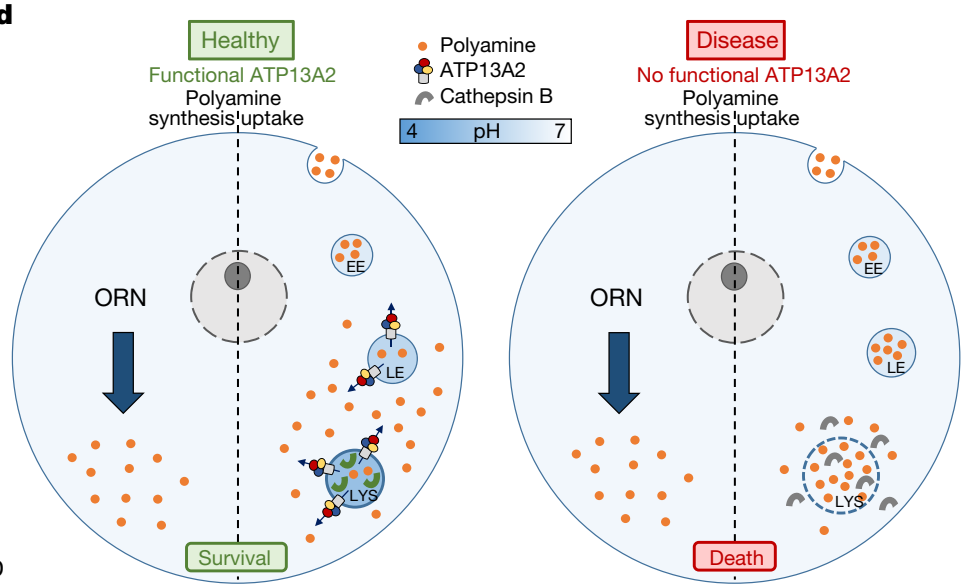

for toxicity. Scale bar, $100 \mu \mathrm{m}$. Top, quantification; bottom: representative images.d, Illustration of the proposed mechanism of endolysosomal polyamine uptake and transfer into the cytosol via ATP13A2. (-), vehicletreated sample; EE, early endosome; LE, late endosome; LYS, lysosome. Data are presented as mean (a) or as box and whisker plots (b, c, horizontal line, median; box boundaries, 25 th and 75 th percentiles) with individual data points representing replicates. The number of independent biological replicates were as follows: $n=2(\mathbf{a}) ; n=3(\mathbf{b}, \mathbf{c})$. Analysis was performed using one-way ANOVA with Tukey's test $(\mathbf{b}, \mathbf{c})$. high concentrations they become toxic ${ }^{18}$. Consistent with this, high levels of SPM or SPD reduced the viability of control cells after 24 hours (Extended Data Fig. 8a, b), which was paralleled by an increase in cell death (Fig. 3a); ornithine and putrescine were not cytotoxic (Extended Data Fig. 8c, d). Notably, loss of ATP13A2 activity exacerbated the toxicity of SPM and SPD (Fig. 3a, Extended Data Fig. 8a, b), which may be a direct consequence of lysosomal polyamine accumulation (Fig. 2d) leading to lysosomal dysfunction. At a time point preceding cell death (4 hours; Extended Data Fig. 8e), lysosomal acidification was compromised in $\mathrm{KO}$ and $\mathrm{KO} / \mathrm{D} 508 \mathrm{~N}$ cells, which was aggravated upon SPM exposure (Fig. 3b). This pH-neutralizing effect of SPM was absent in control and KO/WT cells (Fig. 3b).

Lysosomal alkalization may explain the decreased lysosomaldegradation potential (Fig. 3c) and cathepsin D activity (Extended Data Fig. 8f) that is observed in $\mathrm{KO}$ and KO/D508N cells. However, the activity of cathepsin B increased at toxic SPM levels (Fig. 3d), in line with its higher $\mathrm{pH}$ optimum and most likely due to impaired lysosomal membrane integrity ${ }^{19}$, which is a driver of lysosome-dependent cell death ${ }^{20}$. We then confirmed, using an acridine orange-based assay, that lysosomal membrane integrity in ATP13A2 KO and KO/D508N cells was impaired; this was more prominent after SPM challenge, a phenotype that was absent in control and KO/WT cells (Fig. 3e).SPM treatment also increased the number and size of endogenous galectin 3 punctae, representative of lysosomal rupture, in $\mathrm{KO}$ and $\mathrm{KO} / \mathrm{D} 508 \mathrm{~N}$ cells only (Fig. 3f). Lysosomal rupture was further confirmed by the loss of endolysosomal FITC-dextran punctae and a more diffuse, cytosolic cathepsin B staining in the $\mathrm{KO}$ and $\mathrm{KO} / \mathrm{D} 508 \mathrm{~N}$ cells (Extended Data Fig. $8 g$, h). In line with reported findings ${ }^{21}$, exposure to acidic nanoparticles restored lysosomal $\mathrm{pH}$ and functionality (Fig. 3b, c), prevented cathepsin B activation (Fig. 3d) and recovered the intactness of lysosomal membranes (Fig. 3e), ultimately reducing SPM-induced cell death (Fig. 3g). Moreover, pharmacological inhibition of cathepsin B significantly reduced SPM toxicity in the KO and KO/D508N cells (Fig. 3h).

\section{ATP13A2 is protective in higher disease models}

Lysosomal polyamine toxicity may be relevant in the context of neurodegeneration, because isolated mouse cortical neurons with miRNAmediated Atp13a2 knockdown (Fig. 4a) were more susceptible to SPM-induced cell death than control neurons (Fig. 4b). Notably, the increased sensitivity to SPM observed for neurons in which Atp13a2 was knocked down was attenuated either by inhibition of cathepsin $B$ 
(Extended Data Fig. 9) or by rescue with wild-type human ATP13A2, but not by the D508N mutant (Fig. 4b).

Finally, SPD exposure was found to hamper growth capacity in C. elegans N2 (wild-type); this was marked by developmental delay and hence reduced worm length. These effects were exacerbated in worms deficient in ATP13A2 orthologues (catp-5(O), catp-6(O) or catp-7 $\left.(O)^{22}\right)$ (Fig. 4c). The phenotype of CATP-6- and CATP-7-deficient strains was rescued by wild-type CATP- 6 or CATP-7, but not by a catalytically dead variant, showing that the transport activity is required (Fig. 4c).

\section{Discussion}

Polyamines are physiologically important polycations that are tightly regulated by a complex interplay of import, export, synthesis and degradation ${ }^{18}$. As a polyamine transporter that controls the cellular polyamine content, ATP13A2 emerges as a member of the mammalian polyamine transport system ${ }^{18}$. Extracellular polyamines most probably bind to plasma membrane heparan sulfate proteoglycans ${ }^{23}$ and enter the cell through endocytosis ${ }^{24}$ before transport into the cytosol by ATP13A2 (Fig. 4d). In addition, the other P5B-ATPases (ATP13A3, ATP13A4 and ATP13A5) that reside in the endosomal system share high sequence similarity in the substrate-binding region ${ }^{12}$, and may belong to the mammalian polyamine transport system.

Genetic insights suggest a major role for lysosomal dysfunction in Parkinson's disease, contributing to $\alpha$-synuclein aggregation and mitochondrial dysfunction ${ }^{25}$. Here we demonstrate that impaired lysosomal polyamine export represents a lysosome-dependent cell death pathway that may be implicated in ATP13A2-associated neurodegeneration. In addition, defective ATP13A2 leads to a reduction in cellular polyamine content, which may potentiate the disease phenotype because polyamines are scavengers of heavy metals and reactive oxygen species ${ }^{26}$ and regulate autophagy ${ }^{27}$. The dual effect of ATP13A2 on both lysosomal and cytosolic polyamine levels may explain the broad phenotype that is associated with its loss of function ${ }^{5}$. Other genes that are related to Parkinson's disease may also affect ATP13A2 functionality, or may be affected by disrupted polyamine homeostasis. Polyamine levels decline with age, whereas polyamine supplementation increases lifespan in several model organism ${ }^{27}$. Conversely, defective SPM synthase causes Snyder-Robinson syndrome-a form of X-linked intellectual disability ${ }^{28}$-and reduced expression of SPD/SPM $N^{1}$-acetyltransferase 1 has been implicated in Parkinson's disease ${ }^{29}$. Modulation of polyamine homeostasis may therefore be considered for neuroprotective therapy.

In conclusion, ATP13A2 dysfunction prevents late endolysosomal polyamine export and sensitizes cells to lysosomal disruption by exogenous polyamines.

\section{Online content}

Any methods, additional references, Nature Research reporting summaries, source data, extended data, supplementary information, acknowledgements, peer review information; details of author contributions and competing interests; and statements of data and code availability are available at https://doi.org/10.1038/s41586-020-1968-7.
1. Ramirez, A. et al. Hereditary parkinsonism with dementia is caused by mutations in ATP13A2, encoding a lysosomal type 5 P-type ATPase. Nat. Genet. 38, 1184-1191 (2006).

2. Di Fonzo, A. et al. ATP13A2 missense mutations in juvenile parkinsonism and young onset Parkinson disease. Neurology 68, 1557-1562 (2007).

3. Dehay, B. et al. Loss of P-type ATPase ATP13A2/PARK9 function induces general lysosomal deficiency and leads to Parkinson disease neurodegeneration. Proc. Natl Acad. Sci. USA 109, 9611-9616 (2012).

4. Palmgren, M. G. \& Nissen, P. P-type ATPases. Annu. Rev. Biophys. 40, 243-266 (2011).

5. van Veen, S. et al. Cellular function and pathological role of ATP13A2 and related P-type transport ATPases in Parkinson's disease and other neurological disorders. Front. Mol. Neurosci. 7, 48 (2014).

6. Narayanaswamy, N. et al. A pH-correctable, DNA-based fluorescent reporter for organellar calcium. Nat. Methods 16, 95-102 (2019).

7. De La Hera, D. P., Corradi, G. R., Adamo, H. P. \& De Tezanos Pinto, F. Parkinson's diseaseassociated human $\mathrm{P}_{5 \mathrm{~B}}$-ATPase ATP13A2 increases spermidine uptake. Biochem. J. 450, 47-53 (2013).

8. Heinick, A. et al. Caenorhabditis elegans $\mathrm{P}_{5 \mathrm{~B}}$-type ATPase CATP- 5 operates in polyamine transport and is crucial for norspermidine-mediated suppression of RNA interference. FASEB J. 24, 206-217 (2010).

9. Holemans, T. et al. A lipid switch unlocks Parkinson's disease-associated ATP13A2. Proc. Natl Acad. Sci. USA 112, 9040-9045, (2015).

10. Martin, S., Holemans, T. \& Vangheluwe, P. Unlocking ATP13A2/PARK9 activity. Cell Cycle 14, 3341-3342 (2015).

11. Martin, S. et al. Protection against mitochondrial and metal toxicity depends on functional lipid binding sites in ATP13A2. Parkinsons Dis. 2016, 9531917 (2016).

12. Sørensen, D. M. et al. Parkinson disease related ATP13A2 evolved early in animal evolution. PLoS ONE 13, e0193228 (2018).

13. Vanhoutte, R., Kahler, J. P., Martin, S., van Veen, S. \& Verhelst, S. H. L. Clickable polyamine derivatives as chemical probes for the polyamine transport system. ChemBioChem 19, 907-911 (2018).

14. Demirsoy, S. et al. ATP13A2/PARK9 regulates endo-/lysosomal cargo sorting and proteostasis through a novel PI(3, 5)P2-mediated scaffolding function. Hum. Mol. Genet. 26, 1656-1669 (2017).

15. Seglen, P. O. \& Gordon, P. B. Effects of lysosomotropic monoamines, diamines, amino alcohols, and other amino compounds on protein degradation and protein synthesis in isolated rat hepatocytes. Mol. Pharmacol. 18, 468-475 (1980).

16. Chatr-aryamontri, A. et al. The BioGRID interaction database: 2017 update. Nucleic Acids Res. 45, D369-D379 (2017).

17. Estrada-Cuzcano, A. et al. Loss-of-function mutations in the ATP13A2/PARK9 gene cause complicated hereditary spastic paraplegia (SPG78). Brain 140, 287-305 (2017).

18. Pegg, A. E. Functions of polyamines in mammals. J. Biol. Chem. 291, 14904-14912 (2016).

19. Qiao, C. et al. Atp13a2 deficiency aggravates astrocyte-mediated neuroinflammation via NLRP3 inflammasome activation. CNS Neurosci. Ther. 22, 451-460 (2016).

20. Aits, S. \& Jäättelä, M. Lysosomal cell death at a glance. J. Cell Sci. 126, 1905-1912 (2013).

21. Bourdenx, M. et al. Nanoparticles restore lysosomal acidification defects: implications for Parkinson and other lysosomal-related diseases. Autophagy 12, 472-483 (2016).

22. Zielich, J. et al. Overlapping expression patterns and functions of three paralogous P5B ATPases in Caenorhabditis elegans. PLoS ONE 13, e0194451 (2018).

23. Belting, M. et al. Glypican-1 is a vehicle for polyamine uptake in mammalian cells: a pivital role for nitrosothiol-derived nitric oxide. J. Biol. Chem. 278, 47181-47189 (2003).

24. Uemura, T., Stringer, D. E., Blohm-Mangone, K. A. \& Gerner, E. W. Polyamine transport is mediated by both endocytic and solute carrier transport mechanisms in the gastrointestinal tract. Am. J. Physiol. Gastrointest. Liver Physiol. 299, G517-G522 (2010).

25. Klein, C. \& Westenberger, A. Genetics of Parkinson's disease. Cold Spring Harb. Perspect. Med. 2, a008888 (2012).

26. Ha, H. C. et al. The natural polyamine spermine functions directly as a free radical scavenger. Proc. Natl Acad. Sci. USA 95, 11140-11145 (1998).

27. Madeo, F., Eisenberg, T., Pietrocola, F. \& Kroemer, G. Spermidine in health and disease. Science 359, eaan2788 (2018)

28. Li, C. et al. Spermine synthase deficiency causes lysosomal dysfunction and oxidative stress in models of Snyder-Robinson syndrome. Nat. Commun. 8, 1257 (2017).

29. Lewandowski, N. M. et al. Polyamine pathway contributes to the pathogenesis of Parkinson disease. Proc. Natl Acad. Sci. USA 107, 16970-16975 (2010).

30. Sørensen, D. M., Buch-Pedersen, M. J. \& Palmgren, M. G. Structural divergence between the two subgroups of P5 ATPases. Biochim. Biophys. Acta 1797, 846-855 (2010).

Publisher's note Springer Nature remains neutral with regard to jurisdictional claims in published maps and institutional affiliations.

(c) The Author(s), under exclusive licence to Springer Nature Limited 2020 


\section{Methods}

\section{Data reporting}

No statistical methods were used to predetermine sample size. The experiments were not randomized and the investigators were not blinded to allocation during experiments and outcome assessment.

\section{Materials}

The following reagents were purchased from Sigma-Aldrich: sodium orthovanadate (S6508), $\mathrm{CaCl}_{2}$ (C3881), $\mathrm{ZnCl}_{2}$ (Z0152), $\mathrm{MnCl}_{2}$ (M3634), $\mathrm{FeCl}_{3}$ (157740), SPM (S3256), SPD (S2626), $N^{1}$-acetylspermine trihydrochloride (01467), $N^{1}$-acetylspermidine hydrochloride (9001535-1), $N^{8}$-acetylspermidine dihydrochloride (A3658), putrescine dihydrochloride (P7505), L-arginine (A5006), L-ornithine monohydrochloride (O2375), histamine (H7250), agmatine sulfate salt (A7127), dopamine hydrochloride (H8502), cadaverine (D22606), yeast nitrogen base without amino acids (Y0626), yeast drop-out mix without uracil (Y1501), glucose (G8720), streptavidin sepharose (GE17-5113-01), thrombin (GE27-0846-01), DMSO (276855), DL- $\alpha$-difluoromethylornithine (DFMO; D193), Dynasore (D7693), Pitstop 2 (SML1169), 4-methylumbelliferyl heptanoate (MUH, M2514), propidium iodide (P4170), CA-074 (C5732), fluorescein isothiocyanate-dextran (FITC-dextran, 46945), DAPI (D9542), anti-ATP13A2 antibody (A3361), anti-GAPDH antibody (G8795), Resomer RG 503H (719870) and SigmaFast protease inhibitor (S8820). In addition, 18:1 PtdIns (3,5) $\mathrm{P}_{2}$ (1,2-dioleoyl-sn-glycero-3-phospho-(1'myo-inositol-3',5'-bisphosphate) (ammonium salt); 850154), 18:1 phosphatidic acid (1,2-dioleoyl-sn-glycero-3-phosphate (sodium salt); 840875) and egg phosphatidylcholine (840051) were obtained from Avanti Polar Lipids. Bovine serum albumin (BSA; 3854.3) was obtained from C. Roth. Yeast extract (103753.0500) was purchased from VWR, and $n$-dodecyl- $\beta$-D-maltopyranoside (DDM; 1758-1350) was purchased from Inalco. We obtained Bio-Beads SM-2 resin (1523920) from Bio-Rad. ${ }^{3} \mathrm{H}$-SPM (ART 0471) was ordered from ARC. APCHA ( $\mathrm{N}$-(3-aminopropyl) cyclohexylamine; sc-202715) and 4MCHA (cis-4-methylcyclohexylamine; sc-272662) were purchased from Santa Cruz Biotechnology. Genistein (ab120112), anti-galectin-3 antibody (ab2785), anti-LAMP1 antibody (ab24170) and anti-cathepsin B antibody (ab58802) were purchased from Abcam. TRYPLE (12604021), AO (A1372) and DQ-Green BSA (D12050) were ordered from Life Technologies.

HEK-293T cells were purchased from ATCC and certified by ATCC via STR genotype analysis. SH-SY5Y cells were purchased from ATCC and certified by ATCC via STR genotype analysis. SH-SY5Y cells from an in-house collection were authenticated via DNA fingerprinting (Leibniz-Institut DSMZ-Deutsche Sammlung von Mikroorganismen und Zellkulturen $\mathrm{GmbH}$ ).

\section{Preparation of compounds and inhibitors}

All polyamines, diamines, monoamines and amino acids were prepared to a final stock concentration of $500 \mathrm{mM}(200 \mathrm{mM}$ in the case of SPM) in 0.1 M MOPS-KOH ( $\mathrm{pH} 7.0)$. DFMO was prepared to a final stock concentration of $500 \mathrm{mM}$ in Milli- $\mathrm{QH}_{2} \mathrm{O}$. The inhibitors 4MCHA and APCHA were dissolved in DMSO to a final stock concentration of $200 \mathrm{mM}$. The endocytosis inhibitors Dynasore, genistein and Pitstop 2 were dissolved in DMSO to final concentrations of $50 \mathrm{mM}, 25 \mathrm{mM}$ and $25 \mathrm{mM}$, respectively. The cathepsin B inhibitor CA-074 was dissolved in DMSO to a final concentration of $25 \mathrm{mM}$.

\section{Generation of SH-SY5Y cell models}

SH-SY5Y human neuroblastoma cells were transduced with lentiviral vectors to obtain stable overexpression of firefly luciferase (Fluc) or human ATP13A2 (isoform 2, wild-type (ID: NP_001135445), indicated disease or catalytic mutants) and maintained as described previously ${ }^{9-11}$. The catalytic mutants A467V on M4, D962N on M6 and K1062A on M8 were generated by mutagenesis. P5-type ATPases were discovered by genome sequence analysis 20 years ago and contain highly conserved motifs for function and substrate binding ${ }^{31}$. The A467V mutation converts PPALP of the predicted substrate-binding site in transmembrane segment M4 into PPVLP that is present in ATP13A5 $5^{4,30}$. Also, neighbouring membrane helices contribute to substrate coordination in P-type ATPases, which often relies on conserved and charged residues, such as D962 in M6 and K1062 in M8 of ATP13A2 4 . Furthermore, mutants associated with Kufor-Rakeb syndrome $\left(\mathrm{T} 512 \mathrm{I}^{3,32,33}\right.$ and $\mathrm{G} 872 \mathrm{R}^{34}$ ) or

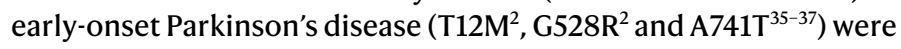
generated. All cell lines were produced at varying viral vector titres and assessed for equal expression to wild-type ATP13A2.

For CRISPR-Cas9-mediated knockout of ATP13A2, the lentiviral vector lentiCRISPRv2 (Addgene, 52961) ${ }^{38}$ was used. First, the Cas9 cassette was transformed into a high-fidelity Cas 9 by Gibson assembly with a gBlock gene fragment (Integrated DNA Technologies) of the Cas9 portion encoding a protein product with high-fidelity mutations (N497A/R661A/Q695A/Q926A). This Cas9 variant triggers fewer offtarget events while retaining its on-target activity ${ }^{39}$. A single-guide RNA (sgRNA) targeted to Atp13a2 was designed taking into account a high on-target efficiency, using sgRNA Designer (https://portals. broadinstitute.org/gpp/public/analysis-tools/sgrna-design $)^{40}$, and a low off-target efficiency, via CRISPR Design (http://crispr.mit.edu/). A lentiviral CRISPR-Cas9 high-fidelity expression plasmid was created by inserting fragments that contained an sgRNA sequence of ATP13A2 (forward, 5'-CACCGGTCAGGGTCCCATAACCGGT; reverse, 5'-AAACACCGGTTATGGGACCCTGACC) into the lentiCRISPRv2 vector. The generated CRISPR-Cas9 high-fidelity ATP13A2 plasmid (1,000 ng), the packaging plasmid pCMV- $\Delta \mathrm{R} 8.91$ (900 ng) and the envelope plasmid pMD2G-VSV-G (Addgene, 12259) (100 ng) were mixed together with $200 \mu \mathrm{l}$ of JetPrime buffer and $4 \mu$ l of JetPrime reagent (Polyplustransfection) for transfection of HEK-293T cells according to the manufacturer's protocol. After $4 \mathrm{~h}$ at $37^{\circ} \mathrm{C}$ and $5 \% \mathrm{CO}_{2}$, the serum-free medium was replaced with DMEM/F12 (Dulbecco's modified Eagle's medium, Nutrient Mixture F-12) supplemented with $10 \%$ fetal calf serum (heat-inactivated). After $48 \mathrm{~h}$, the lentiviral vectors were collected by passing the medium through a $0.45-\mu \mathrm{m}$ filter, and $0.5 \mathrm{ml}$ of this medium was used to transduce SH-SY5Y cells supplemented with 8 $\mu \mathrm{g} \mathrm{ml}^{-1}$ polybrene (Sigma-Aldrich). $24 \mathrm{~h}$ after transduction, cells were selected in $3 \mu \mathrm{g} \mathrm{ml}^{-1}$ puromycin (Sigma-Aldrich) and passaged three times before single clones were isolated via serial dilution. The resulting cells were examined by quantitative PCR (qPCR) (ATP13A2 forward, 5'-ACCGGTTATGGGACCCTGAC; ATP13A2 reverse, 5'-GTGATAGCCGA TGACCCTCC) with HPRT and TBP as internal controls (HPRT forward, 5'-TGAGGATTTGGAAAGGGTGTTT; HPRT reverse, 5' -ACATCTCGA GCAAGACGTTCAG; TBP forward, 5'-CGGCTGTTTAACTTCGCTTC; $T B P$ reverse, $5^{\prime}$-CACACGCCAAGAAACAGTGA) and western blotting. For rescue experiments, ATP13A2 knockout cells were stably transduced with lentiviral vectors expressing wild-type ATP13A2 or the D508N mutant in which both cDNAs were modified with synonymous mutations at the sgRNA target site. All cell lines were routinely assessed for mycoplasma and cultured for a maximum of 20 passages.

\section{Membrane fractionation}

SH-SY5Y cells were seeded in $15-\mathrm{cm}$ dishes at a density of $6 \times 10^{6}$ cells per plate. The cells were collected $24 \mathrm{~h}$ later after trypsinization and brief centrifugation $(300 \mathrm{~g}, 5 \mathrm{~min})$. Subcellular fractionation was performed by differential centrifugation, as described previously ${ }^{9-11}$. The microsomal protein concentration was measured using the bicinchoninic acid assay (Thermo Fisher Scientific, Pierce) according to the manufacturer's instructions.

\section{ATPase assay}

The ATPase activity of ATP13A2 was assessed using a commercially available luminescence assay (ADP-Glo Max assay, Promega) that monitors the production of ADP via luciferase activity. Substrate screen was designed to include candidates previously postulated in 
the literature $\mathrm{e}^{6-8,41-45}$. The reactions were performed for $30 \mathrm{~min}\left(37^{\circ} \mathrm{C}\right)$ in a final volume of $25 \mu \mathrm{l}$. The assay reaction mixture contained $50 \mathrm{mM}$ MOPS-KOH (pH 7), 100 mM KCl, 11 mM MgCl, 1 mM DTT, $195 \mu$ M DDM, various concentrations of the indicated compound, and either microsomes $(5 \mu \mathrm{g})$ collected from SH-SY5Y cells overexpressing ATP13A2 (wild-type or mutants) or purified ATP13A2 (0.3-0.5 $\mu \mathrm{g})$. When purified ATP13A2 was used, we included $125 \mu \mathrm{M}$ phosphatidic acid, $125 \mu \mathrm{M}$ PtdIns $(3,5) \mathrm{P}_{2}$ and $19.5 \mu \mathrm{M}$ DDM in the reaction buffer. The assay was started by the addition of $5 \mathrm{mM}$ ATP and was terminated by adding $25 \mu$ l of ADP-Glo Reagent. The 96-well plate was then incubated for 40 min at room temperature, followed by the addition of $50 \mu$ l of ADP-Glo Max Detection Reagent. After 40 min, luminescence was detected using a FlexStation 3.0 system (Molecular Devices). Dose-response curves and $n, K_{\mathrm{m}}$ and $V_{\max }$ values were calculated using GraphPad Prism Software (GraphPad Software).

\section{Autophosphorylation assay}

The autophosphorylation activity of ATP13A2 on the conserved D508 residue was measured as described previously,12,17. In brief, microsomes $(20 \mu \mathrm{g})$ or purified ATP13A2 $(1 \mu \mathrm{g})$ were incubated with radioactively labelled ATP in the presence of the indicated SPM or ornithine concentrations, and after $1 \mathrm{~min}$ the reaction was stopped. In the case of purified ATP13A2, $125 \mu \mathrm{M}$ phosphatidic acid and $125 \mu \mathrm{M}$ PtdIns $(3,5)$ $\mathrm{P}_{2}$ were included in the reaction mixture. To determine the sensitivity of the ATP13A2 phosphoenzyme to ATP or a combination of ATP and SPM, 30 s after adding ${ }^{32} \mathrm{P}$-ATP samples were incubated with nonradioactive ATP (5 $\mathrm{mM}$ in experiments using microsomes, $1 \mathrm{mM}$ for purified ATP13A2) and SPM (1 mM) before the reaction was stopped at the indicated time points. The incorporation of ${ }^{32} \mathrm{P}$ was visualized after SDS-PAGE under acidic conditions and subsequently detected by autoradiography (microsomes) or liquid scintillation counting (purified ATP13A2) (Liquid Scintillation Analyzer TRI-CARB 2900TR).

\section{Transformation and overexpression of ATP13A2 in yeast}

The S. cerevisiae W303-1B/Gal4- $\Delta$ Pep4 strain (leu2-3, his3-11,15, trp11::TRP1-GAL10-GAL4, ura3-1, ade2-1, canr, cir $^{+}, \Delta$ Pep4 MAT; a gift from R. Lopez Marques) was transformed according to the lithium acetate/ single-stranded carrier DNA/polyethylene glycol method ${ }^{46}$, with the pYeDP60 vector containing a yeast codon-optimized version of human $A T P 13 A 2$ variant 2 (wild-type or the catalytically dead E343A mutant) followed by a thrombin cleavage site and a C-terminal BAD tag ${ }^{47,48}$. The transformation mixture was grown for $48 \mathrm{~h}$ at $30^{\circ} \mathrm{C}$ on minimal medium agar plates lacking uracil $(0.54 \%$ yeast nitrogen base without amino acids, $0.12 \%$ yeast drop-out mix without uracil, $2 \%$ glucose and $2 \%$ agar) to select yeast colonies that acquired the plasmid. These colonies were then cultured in $20 \mathrm{ml}$ of MM-Ura medium $(0.67 \%$ yeast nitrogen base without amino acids, $0.19 \%$ yeast drop-out mix without uracil, and $2 \%$ glucose) and grown for $24 \mathrm{~h}$ at $28^{\circ} \mathrm{C}$ and $200 \mathrm{rpm}$. The MM-Ura yeast pre-culture was used to inoculate $100 \mathrm{ml}$ of MM-Ura medium to a final $\mathrm{OD}_{600}$ of 0.2 , followed by a 12 -h incubation period $\left(28^{\circ} \mathrm{C}\right.$ and $\left.200 \mathrm{rpm}\right)$. The second pre-culture was inoculated into 4.51 of YPGE2X medium ( $2 \%$ yeast extract, $2 \%$ bactopeptone, $1 \%$ glucose and $2.7 \%$ ethanol) to a final $\mathrm{OD}_{600}$ of 0.05 and grown for $36 \mathrm{~h}\left(28^{\circ} \mathrm{C}\right.$ and $175 \mathrm{rpm})$. ATP13A2-BAD expression was induced with $2 \%$ galactose, followed by a second galactose induction $12 \mathrm{~h}$ later. After another $12 \mathrm{~h}$, the pellet was collected $\left(1,000 \mathrm{~g}, 10 \mathrm{~min}, 4^{\circ} \mathrm{C}\right)$.

\section{Yeast membrane preparation}

Yeast cells were broken with glass beads using a BeadBeater (BioSpec products). The lysis buffer contained $50 \mathrm{mM}$ Tris $-\mathrm{HCl}$ (pH 7.5), $1 \mathrm{mM}$ EDTA, $0.6 \mathrm{M}$ sorbitol, $1 \mathrm{mM}$ phenylmethylsulfonyl fluoride and SigmaFast protease inhibitor. To remove cell debris and nuclei, the crude extract was centrifuged at $2,000 \mathrm{~g}$ for $20 \mathrm{~min}\left(4^{\circ} \mathrm{C}\right)$. The supernatant (S1) was centrifuged at $20,000 \mathrm{~g}$ for $20 \mathrm{~min}\left(4^{\circ} \mathrm{C}\right)$ to pellet the heavy membrane fraction (P2), and the resulting supernatant (S2) was further centrifuged at $200,000 \mathrm{~g}$ for $1 \mathrm{~h}\left(4^{\circ} \mathrm{C}\right)$. The resulting pellet (that is, the light membrane fraction, $\mathrm{P} 3$ ) was resuspended in $20 \mathrm{mM}$ HEPES-Tris ( $\mathrm{pH} 7.4), 0.3 \mathrm{M}$ sucrose and $0.1 \mathrm{mM} \mathrm{CaCl}_{2}$. The total protein concentration was determined using a Bradford assay (B6916, Sigma-Aldrich).

\section{Purification of ATP13A2-BAD}

The method used to purify ATP13A2 was based on the purification of overexpressed Drs2p from yeast membranes ${ }^{47}$. Yeast P3 membranes were diluted to $5 \mathrm{mg}$ of total protein per $\mathrm{ml}$ in SSR buffer ( $50 \mathrm{mM}$ MOPS$\mathrm{KOH}$ (pH 7), $100 \mathrm{mM} \mathrm{KCl}, 20 \%$ glycerol, $5 \mathrm{mM} \mathrm{MgCl}_{2}, 1 \mathrm{mM} \mathrm{DTT}$, and SigmaFast protease inhibitor cocktail) and solubilized using DDM, with a detergent-to-protein ratio of 1:1. The samples were stirred on ice for $30 \mathrm{~min}$, followed by centrifugation $\left(100,000 \mathrm{~g}, 1 \mathrm{~h}, 4^{\circ} \mathrm{C}\right)$ to pellet non-solubilized membranes. The solubilized material was incubated with streptavidin beads for $4 \mathrm{~h}\left(4^{\circ} \mathrm{C}\right)$ to enable binding of BAD-tagged ATP13A2 to the resin. To eliminate unbound material, the resin was washed four times with three resin volumes of SSR buffer supplemented with $0.5 \mathrm{mg} \mathrm{ml}^{-1} \mathrm{DDM}$. Subsequent cleavage by thrombin $(0.0625 \mathrm{U}$ per mg total protein) enabled the release of ATP13A2 from the beads by overnight incubation at $4{ }^{\circ} \mathrm{C}$. Finally, a Vivaspin Turbo 4 concentrator (100 kDa molecular weight cut-off, Sartorius) was used to concentrate the sample. The protein concentration was determined using a Bradford assay. The quality of the purification was evaluated via SDS-PAGE followed by Coomassie staining or immunoblotting, as described previously $^{9-11}$. Furthermore, the purified ATP13A2 sample was analysed by linear mode MALDI-TOF MS (matrix-assisted laser desorption timeof-flight mass spectrometry; Applied Biosystems 4800 Proteomics Analyzer) in the presence of $\alpha$-cyano-4-hydroxycinnamic acid as matrix and after C4 omix (Agilent) pipette tip purification.

\section{Reconstitution of yeast membranes}

To reconstitute yeast membranes, we followed a similar strategy as described before ${ }^{49}$.P3 membranes from the yeast membrane preparation expressing the ATP13A2-BAD construct were solubilized in buffer T (10 mM Tris- $\mathrm{HCl}$ ( $\mathrm{pH} 7.4$ ) and $1 \mathrm{mMEDTA}$ ) supplemented with 1.4\% DDM. After removing the insoluble fraction by ultracentrifugation $(30 \mathrm{~min}$, $200,000 \mathrm{~g}$ ), the detergent extract was supplemented with $4.5 \mathrm{mM}$ egg phosphatidylcholine and $0.5 \mathrm{mM} 18: 1$ phosphatidic acid (in buffer $\mathrm{T}$ containing 0.7\% DDM). The extract was then treated with Bio-Beads to remove the DDM and reconstitute proteoliposomes (that is, 'no ATP inside' condition). To generate proteoliposomes that contained intraluminal ATP (that is, 'ATP inside' condition), we added $5 \mathrm{mM}$ ATP and an ATP-regenerating system before incubation with the Bio-Beads. Finally, the vesicles were recovered by ultracentrifugation $(1 \mathrm{~h}, 200,000 \mathrm{~g})$ and resuspended in buffer $\mathrm{T}$. The protein concentration was determined using a Bradford assay.

\section{Transport assay using reconstituted vesicles}

Uptake of ${ }^{3} \mathrm{H}$-SPM into freshly prepared vesicles was measured within 60 min. The above-described vesicles ('no ATP inside' or 'ATP inside') were

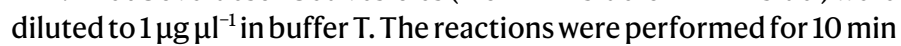
$\left(30^{\circ} \mathrm{C}\right)$ in a final volume of $1 \mathrm{ml}$. The assay reaction mixture contained $50 \mathrm{mM}$ MOPS, $100 \mathrm{mM} \mathrm{KCl}^{11} \mathrm{mM} \mathrm{MgCl}_{2}, 1 \mathrm{mMDTT}$ and reconstituted vesicles $(45 \mu \mathrm{g})$. The reaction was started by adding $1 \mathrm{mM}^{3} \mathrm{H}$-SPM. For the condition 'no ATP inside', $5 \mathrm{mM}$ ATP and an ATP-regenerating system were added after Bio-Bead treatment, before the addition of ${ }^{3} \mathrm{H}$-SPM. The reaction was stopped by filtering the samples through Millipore filters $(0.45 \mu \mathrm{m})$. After washing of the filters with assay buffer, radioactivity retained on the filters was counted using a liquid scintillation counter (Liquid Scintillation Analyzer TRI-CARB 2900TR).

\section{Cellular transport assay and endocytosis assessment}

BODIPY-SPD and BODIPY-SPM were synthesized as described previously (compounds 14 and 15 , respectively) ${ }^{13}$. The cells were seeded in 12 -well plates $\left(1.0 \times 10^{5}\right.$ cells per well) and the next day the cells were 
incubated with $5 \mu \mathrm{M}$ BODIPY-SPM or BODIPY-SPD for $2 \mathrm{~h}$ before collection. To assess endocytosis, the cells were pre-treated ( $30 \mathrm{~min})$ with the endocytosis inhibitors Dynasore $(100 \mu \mathrm{M})$, genistein $(50 \mu \mathrm{M})$ and/or Pitstop $2(50 \mu \mathrm{M})$ before the addition of either $20 \mu \mathrm{g} \mathrm{ml}^{-1}$ FITC-dextran or BODIPY-SPM $\left(2 \mathrm{~h}\right.$ and $\left.37^{\circ} \mathrm{C}\right)$. The cells were then collected $(300 \mathrm{~g}$, $5 \mathrm{~min})$, washed and resuspended in PBS containing $1 \%$ BSA. Finally, an Attune Nxt (Thermo Fisher Scientific) flow cytometer was used to record the mean fluorescence intensities (MFI) of 10,000 events per treatment.

\section{Metabolomics}

Cells were grown into a 6-well plate and extracted as described previously $^{50}$. In brief, the medium was removed and cells were washed with a $0.9 \% \mathrm{NaCl}$ solution. The washing solution was removed and $150 \mu \mathrm{l}$ of a $6 \%$ trichloroacetic acid (Sigma) was added for the extraction. Using a cell scraper, the full extract was transferred into an eppendorf and incubated for $30 \mathrm{~min}$ on ice. Insoluble material, such as precipitated proteins, was removed by centrifugation for $20 \mathrm{~min}$ at $20,000 \mathrm{~g}$ at $4{ }^{\circ} \mathrm{C}$. To $100 \mu \mathrm{l}$ of the supernatant, $900 \mu \mathrm{l}$ of a $100 \mathrm{mM}$ sodium carbonate buffer (pH 9.0) was added. Next, $25 \mu \mathrm{l}$ of isobutyl chloroformate (Sigma) was added and the mixture was incubated for $30 \mathrm{~min}$ at $35^{\circ} \mathrm{C} .800 \mu \mathrm{l}$ of the reaction mixture was transferred to a $2 \mathrm{ml}$ eppendorf tube and $1 \mathrm{ml}$ of diethylether (Sigma) was added. The mixture was vortexed vigorously and placed for $15 \mathrm{~min}$ at $25^{\circ} \mathrm{C} .900 \mu \mathrm{l}$ of the upper phase was transferred into an eppendorf and dried using a vacuum centrifuge. Finally, the dried extract was dissolved in $125 \mu$ of a $50 \%$ acetonitrile (LC-MS grade, Merck) solution in water containing $0.2 \%$ acetic acid.

$15 \mu$ l of the extract was loaded onto a Thermo Scientific Liquid Chromatography QQQ (Quantiva, Thermo Fisher Scientific) equipped with an ACQUITY UPLC BEH C18 $(1.7 \mu \mathrm{m}, 2.1 \times 100 \mathrm{~mm})$ column from WATERS. Solvent A consisted of ultrapure $\mathrm{H}_{2} \mathrm{O}$ with $0.2 \%$ acetic acid while solvent B was acetonitrile (Merck) with $0.2 \%$ acetic acid; all solvents used were LC-MS grade. Flow rate remained constant at $250 \mu \mathrm{min}^{-1}$, and the column temperature remained constant at $30^{\circ} \mathrm{C}$. A gradient for the separation of modified polyamines was applied as follows: from 0 to $2 \mathrm{~min} 20 \% \mathrm{~B}$, from 2 to $10 \mathrm{~min}$ a linear increase to $85 \%$ B was carried out and $85 \%$ B was maintained until 17 min. At $18 \mathrm{~min}$ the gradient returned to $20 \% \mathrm{~B}$. The method stopped at $22 \mathrm{~min}$. The mass spectrometer operated in positive ion mode $(3,500 \mathrm{~V})$; the source settings were as follows: sheath gas at 50 , aux gas at 10 , the ion transfer tube was heated at $325^{\circ} \mathrm{C}$ and the vaporizer temperature was set at $350^{\circ} \mathrm{C}$. The mass spectrometer operated in multiple reaction monitoring mode and used the following transitions: putrescine (parent $m / z$ at $289.2 \rightarrow$ fragment 215.2 , collision energy at $10.25 \mathrm{~V}$ ), SPD (parent $m / z$ at $446.4 \rightarrow$ fragment 198.2 , collision energy at $23.6 \mathrm{~V}$ ) and SPM (parent $m / z$ at $603.4 \rightarrow$ fragment 455.1 , collision energy at $19.91 \mathrm{~V}$ ). Peak area was integrated using the XCalibur Quan tool (version 4.2.28.14, Thermo Fisher Scientific).

\section{Preparation and characterization of acidic nanoparticles}

Acidic nanoparticles were prepared as previously described ${ }^{21}$. In brief, $31 \mathrm{mg}$ of Resomer RG 503H (lactide to glycolide ratio 50:50, molecular weight $24-38 \mathrm{kDa}$ ) was dissolved in $3.1 \mathrm{ml}$ of tetrahydrofuran and subsequently $200 \mu \mathrm{l}$ of this solution was added to $20 \mathrm{ml}$ of ultrapure water under sonication. The suspension was then concentrated using the rotary evaporator to a final volume of approximately $12 \mathrm{ml}$, resulting in a concentration of $0.167 \mathrm{mg} \mathrm{ml}^{-1}$. The size distribution of prepared nanoparticles was measured using a Wyatt DynaPro DLS plate reader (Wyatt), using an 830-nm laser in a flat-bottom 384-well plate (Greiner) at $25^{\circ} \mathrm{C}$ and 10 measurements were averaged per experiment.

\section{MUH cytotoxicity assay}

SH-SY5Y cells were seeded in 96 -well plates $\left(1 \times 10^{4}\right.$ cells per well $)$ and allowed to adhere overnight. The cells were subsequently treated with increasing doses of the indicated compounds for $24-48 \mathrm{~h}$. After exposure, the cells were washed with PBS and stained with $300 \mu \mathrm{g} \mathrm{ml}^{-1}$ MUH (prepared in DMSO, dissolved in PBS) for 30 min at $37^{\circ} \mathrm{C}$. Cytotoxicity was read using a FlexStation 3.0 multi-well plate reader (Molecular Devices; excitation 360 nm, emission 460 nm, cut-off 455 nm). Data were expressed relative to control.

\section{Propidium iodide exclusion assay}

Cells were seeded in 12 -well plates $\left(1.0 \times 10^{5}\right.$ cells per well $)$ and the next day the cells were treated with increasing doses of SPM, alone or in combination with the cathepsin B inhibitor CA-074 $(25 \mu \mathrm{M}, 1 \mathrm{~h}$ preincubation) or acidic nanoparticles (180 $\mathrm{ng} \mathrm{ml}^{-1}, 1 \mathrm{~h}$ pre-incubation), and incubated for $24 \mathrm{~h}$ at $37^{\circ} \mathrm{C}$. Thereafter, the cells were collected following trypsinization and a brief centrifugation ( $300 \mathrm{~g} ; 5 \mathrm{~min})$, washed with PBS and stained with $1 \mu \mathrm{g} \mathrm{ml}^{-1}$ propidium iodide (in PBS containing 1\% BSA). An Attune Nxt (Thermo Fisher Scientific) flow cytometer was used to determine the proportion of propidium iodide-positive cells $(10,000$ events per treatment).

\section{FITC-dextran-based lysosomal pH}

The protocol was adapted from refs. ${ }^{51,52}$.SH-SY5Y cells were seeded in 12 -well plates $\left(1.0 \times 10^{5}\right.$ cells per well $)$ and allowed to adhere overnight. Cellswereexposed to $50 \mu \mathrm{g} \mathrm{ml}^{-1} \mathrm{FITC}$-dextran for $72 \mathrm{~h}$. Sampleswere then washed and placed in fresh medium for $2 \mathrm{~h}$ before treatment with SPM $(10 \mu \mathrm{M})$ alone or in combination with acidic nanoparticles $\left(180 \mathrm{ng} \mathrm{ml}^{-1}\right.$, $1 \mathrm{~h}$ pre-incubation) for a further $4 \mathrm{~h}$. Samples were then collected by centrifugation $(300 \mathrm{~g}, 5 \mathrm{~min}$ ) and washed in PBS. Cells were finally resuspended in $500 \mu \mathrm{l}$ of PBS containing $1 \% \mathrm{BSA}$ and FITC dual emission was assessed by flow cytometry (excitation $488 \mathrm{~nm}$, emission $530 \mathrm{~nm}$ (BL1) and $600 \mathrm{~nm}$ (BL2)) of 10,000 events per condition using an Attune NXT flow cytometer (Thermo Fisher Scientific). The emission ratio (BL1/ BL2) of all samples were compared to a standard curve, whereby signals were obtained from untreated cells resuspended in monensin (100 $\mu \mathrm{M})$ containing Britton Robinson buffer with increasing $\mathrm{pH}$ (3.0-8.0).

\section{Lysosomal degradative capacity}

Cell lines were seeded in 12 -well plates $\left(1.0 \times 10^{5}\right.$ cells per well $)$ and the next day the cells were pre-treated with SPM $(10 \mu \mathrm{M})$ for $1 \mathrm{~h}$ at $37^{\circ} \mathrm{C}$. For samples requiring exposure to acidic nanoparticles, cells were treated with $180 \mathrm{ng} \mathrm{ml}^{-1} 1 \mathrm{~h}$ before the addition of SPM. Subsequently, $5 \mu \mathrm{g} \mathrm{ml}^{-1} \mathrm{DQ}$-Green BSA was added to the cells for a further $3 \mathrm{~h}\left(37^{\circ} \mathrm{C}\right)$. Finally, the cells were collected $(300 \mathrm{~g}, 5 \mathrm{~min})$, and the MFI of 10,000 events were assessed using an Attune Nxt (Thermo Fisher Scientific) flow cytometer.

\section{Cathepsin activity assays}

SH-SY5Y cells were seeded in $10-\mathrm{cm}$ plates $\left(2 \times 10^{6}\right.$ cells per plate $)$ and allowed to adhere overnight before treatment with $10 \mu \mathrm{MSPM}$ for $4 \mathrm{~h}$ at $37^{\circ} \mathrm{C}$. For samples requiring the addition of acidic nanoparticles, $180 \mathrm{ng} \mathrm{ml}^{-1}$ nanoparticles were added $1 \mathrm{~h}$ before the addition of SPM. Next, the samples were collected using TRYPLE and a brief centrifugation (300g, 5 min). The activities of cathepsin B (ab65300) and cathepsin D (ab65302) were assessed using commercially available kits (Abcam) according to the manufacturer's instructions. MFI values were acquired using a FlexStation 3.0 multi-well plate reader (Molecular Devices).

\section{Lysosomal membrane integrity}

SH-SY5Y cells were seeded in 12 -well plates $\left(1.0 \times 10^{5}\right.$ cells per well $)$ and the next day the cells were incubated with $5 \mu \mathrm{g} \mathrm{ml}^{-1}$ acridine orange (dissolved in medium) for $15 \mathrm{~min}$ at $37^{\circ} \mathrm{C}$. Thereafter, the medium was discarded, the cells were washed, and fresh medium was added. For samples requiring acidic nanoparticles, cells were treated with $180 \mathrm{ng} \mathrm{ml}^{-1} 1 \mathrm{~h}$ before the addition of SPM. The cells were then treated with $10 \mu \mathrm{M} \mathrm{SPM}$ for $4 \mathrm{~h}$ at $37^{\circ} \mathrm{C}$. Finally, the cells were collected and resuspended in PBS containing $1 \%$ BSA. The MFI of 10,000 events was 
captured using an Attune Nxt (Thermo Fisher Scientific) flow cytometer.

\section{BODIPY-SPM localization and lysosomal rupture analysis}

For all immunofluorescent stainings, SH-SY5Y cell lines were seeded in 12 -well plates $\left(0.25 \times 10^{5}\right.$ cells per well on coverslips $)$ and the next day the cells were incubated with the indicated compounds for $4 \mathrm{~h}$ at $37^{\circ} \mathrm{C}$. For BODIPY-SPM analysis, cells were pulsed with $5 \mu \mathrm{M}$ BODIPY-SPM for $15 \mathrm{~min}$, washed and chased in fresh medium for a further $105 \mathrm{~min}$ at $37^{\circ} \mathrm{C}$. After treatment, the cells were washed twice in PBS, fixed in $4 \%$ paraformaldehyde for $30 \mathrm{~min}\left(37^{\circ} \mathrm{C}\right)$, washed in PBS and stored at $4^{\circ} \mathrm{C}$. For immunofluorescence staining, the cells were washed in PBS containing $0.5 \%$ Tween 20 (PBS-T), permeabilized in PBS containing $0.1 \%$ Triton X-100 (30 min) and blocked first in $0.1 \mathrm{M}$ glycine $(1 \mathrm{~h})$ and then in PBS-T containing $1 \%$ fetal calf serum and $10 \%$ BSA (30 min). Gal3-, LAMP1- and cathepsin B-specific antibodies were used at a dilution of 1:100-200 (in PBS-T containing $1 \%$ BSA) overnight at $4{ }^{\circ} \mathrm{C}$. Subsequently, the samples were washed and incubated with Alexa Fluor secondary antibody (1:1,000;30 min). To assess FITC-dextran release, cells were loaded with $50 \mathrm{\mu g} \mathrm{ml}^{-1} \mathrm{FITC}$-dextran for $72 \mathrm{~h}$, cells were then washed and placed in fresh medium for $1 \mathrm{~h}$ before the addition of SPM $(10 \mu \mathrm{M})$. To visualize the nucleus, all samples were stained with DAPI $\left(200 \mathrm{ng} \mathrm{ml}^{-1}\right.$, $15 \mathrm{~min})$. After staining, the samples were fixed to slides, and images were acquired using an LSM780 or LSM880 confocal microscope (Zeiss). For the acquisition of BODIPY-SPM, images were taken with equal settings to confirm uptake potential of ATP13A2 (Extended Data Fig. 1i). To assess the intracellular distribution of BODIPY-SPM (Fig. 2d, Extended Data Fig. $1 j-1)$, microscope settings were optimized per cell type to enable a comparable assessment of BODIPY-SPM localization in KO/WT and $\mathrm{KO} / \mathrm{D} 508 \mathrm{~N}$ cells.

\section{Neuron isolation}

Primary cortical neurons were derived from FVB/N mice embryos at embryonic day (E)16. Pregnant mice were euthanized on gestation day 16 by cervical dislocation. The brains of E16 mouse pups were collected and placed in a dish containing calcium- and magnesium-free Hanks' Balanced Salt Solution (HBSS, Life Technologies, 14180-046) on ice. Both cerebral hemispheres were separated from the cerebellum. Meninges were removed from the cerebral hemispheres and the brain cortices dissected. Brain cortices were collected, washed twice and digested with $0.05 \%$ trypsin (Life Technologies, 25300-054, $10 \mathrm{~min}$ at $37^{\circ} \mathrm{C}$ ). The trypsin reaction was terminated by the addition of $7 \mathrm{ml}$ HBSS and $1 \mathrm{ml}$ of horse serum. Cells were separated by pipetting and filtration through a cell strainer $(40 \mu \mathrm{m}$, Falcon, 352340). Cells were centrifuged at $1,000 \mathrm{rpm}$ for $5 \mathrm{~min}\left(4^{\circ} \mathrm{C}\right)$, the supernatant discarded and the pellet suspended in $5 \mathrm{ml}$ Dulbecco's modified Eagle's medium (DMEM; Sigma-Aldrich, D6546) containing GlutaMAX (Life Technologies, 31966-021), 5\% horse serum (Life Technologies, 26050-088) and $20 \mathrm{mM}$ glucose (Sigma-Aldrich, 8270). Primary cortical neurons were plated in 12-well plates, on coverslips coated with poly-D-lysine (Sigma-Aldrich, P6407). After an overnight incubation, cell medium was exchanged for Neurobasal medium (Life Technologies, 21103-049) supplemented with 2 mM L-glutamine (Life Technologies, 25030-24) and 2\% B27 (Life Technologies, 17504-044).

\section{Atp13a2 knockdown and rescue in isolated cortical neurons}

For knockdown, microRNA (miR)-based short-hairpin lentiviral vectors were generated as described ${ }^{53}$. The two most potent miRs against mouse Atp13a2 (mouse miR-3, CCACGCCGAAACACTCGTTATA and mouse miR-5, CGCCGAAACACTCGTTATAGAA) were used to induce knockdown in mouse primary neurons. A miR targeting Fluc was used as a control (miR-Fluc, ACGCTGAGTACTTCGAAATGTC). Primary neurons were transduced 4 days after isolation, $72 \mathrm{~h}$ before experimentation. For rescue of ATP13A2 expression in knockdown conditions, neurons were subjected to a second round of transductions with either human wild-type or the D508N variant of ATP13A2, $24 \mathrm{~h}$ after the addition of the miR. Fluc was used as an overexpression control. At day 7 post-isolation, cells were treated with $10 \mu \mathrm{M} \mathrm{SPM}$ for $24 \mathrm{~h}$. To test the contribution of cathepsin B to SPM-induced cell death, neurons were pre-treated (30 min) with $10 \mu \mathrm{MCA}-074$. Knockdown efficiency was validated with qPCR on mRNA levels $72 \mathrm{~h}$ after transduction using the following primers (Atp13a2 forward, CATGGCCCTCTACAGCCTGA; Atp13a2 reverse, CTCATGAGCACCGCAACCGT) with Gapdh as internal control (forward, TGTGTCCGTCGTGGATCTGA; reverse, CCTGCTTCACCACCTTCTTGA). All mouse primary neuron experiments were carried out in accordance with the European Communities Council Directive of November 24 1986 (86/609/EEC) and approved by the Bioethical Committee of the KU Leuven (Belgium) (ECD project P185-2014).

\section{TUNEL staining}

TUNEL staining was assessed according to the manufacturer's protocol for the Click-iT Plus TUNEL assay (Thermo Fisher Scientific, C10617). DAPI was used as a nuclear counterstain and images were acquired using a LSM780 confocal microscope.

\section{Caenorhabditis elegans}

During routine culture, nematodes were grown on NGM (nematode growth medium) using Escherichia coli strain AMA1004 as food source $^{54,55}$. In order to reduce the likelihood that bacterial catabolism and divalent cations would interfere with SPD assays, these were done using DCDA (divalent-cation-depleted agar) plates, which do not permit bacterial growth. This medium contains $2 \%$ agar (Carl Roth 5210) that has been washed with $50 \mathrm{mM}$ EDTA, followed by multiple washes with reverse osmosis-purified water; 50 mM HEPES pH 7.4; and $20 \mu \mathrm{g} \mathrm{ml}^{-1}$ kanamycin. SPD trihydrochloride (1 M stock solution; Sigma S2501) was added to a final concentration of $5 \mathrm{mM}$ after microwaving to melt the agar. Assays were performed in 35-mm plates that contained $1 \mathrm{ml}$ of DCDA. Approximately $2 \mu \mathrm{l}$ of $E$. coli was transferred to each assay plate from the lawn of a seeded NGM plate. In each case, multiple adult hermaphrodites were added to the assay plate and allowed to lay eggs for $8 \mathrm{~h}$ (datasets 1 and 3) or $15 \mathrm{~h}$ (dataset 2) at $23.5^{\circ} \mathrm{C}$, then removed. Incubation of plates at $23.5^{\circ} \mathrm{C}$ was continued until the $0 \mathrm{mM}$ SPD N2 plates contained many mid-late stage $L 4 \mathrm{~s}$, at which point worms were rinsed off the plates and stored in microfuge tubes at $-20^{\circ} \mathrm{C}$. Worm lengths were determined by mounting the thawed (dead) animals on an agarose pad covered by a coverslip, capturing images using a Leica M205 FA microscope equipped with digital camera plus software, and measuring the length of each worm from snout to tail tip using Image J.

C. elegans expresses three ATP13A2 orthologues: CATP-5, CATP-6 and CATP-7. Therefore, in this study the following mutant alleles were used, each of which was backcrossed to N2 Bristol at least three times: catp-6(ok3473) IV, catp-7(tm4438) IV, catp-5(tm4481) $\mathrm{X}^{56}$. Each of these is a null allele, so they are referred to in the text as catp-\# $(O)$.

Transgenic strains carrying extrachromosomal arrays with the pRF4 rol-6(su1006) plasmid were generated by microinjection and maintained as described previously ${ }^{57}$. Injection mixes typically contained $100 \mu \mathrm{g} \mathrm{ml}^{-1} \mathrm{pRF} 4$, plus $30 \mu \mathrm{g} \mathrm{ml}^{-1}$ of the test construct. In some cases, Pmyo-2::gfp was included at approximately $5 \mathrm{\mu g} \mathrm{ml}^{-1}$ as an additional method for detecting transgenic animals. To generate transport-defective versions of catp-6 and catp-7, plasmids carrying catp-6::mKate2 and catp-7::GFP ${ }^{22}$ were modified to change the coding sequence for the conserved DKTGT autophosphorylation motif to NKTGT. Proper expression and subcellular localization of CATP-6::mKate2 and CATP-7::GFP was verified in the case of all transgenic strains that were used. (catp-6(0); [catp-6(+)]) and catp-7(O);[catp-7(+)] express CATP-6 and wild-type CATP-7 in the null background, respectively; catp-6(O); [catp-6(D465N)] and catp$7(0) ;[$ catp-7(D5O3N)] express CATP-6(D465N) and CATP-7(D503N) in the null background). 


\section{Statistics and reproducibility}

Data are expressed as the mean \pm s.e.m. or with individual data points (replicates of multiple independent experiments) shown on group means or box and whisker plots (indication of median, 25th percentile, 75 th percentile and minimum to maximum value range). Flow cytometry was set up and gated as described in Supplementary Fig. 2. GraphPad Prism 7.04 was used to plot all graphs and to perform all of the required statistical and quantitative assessments. Statistical tests for each graph are described in the legend. The number of independent biological experiments for each panel is highlighted in the figure legends. For the cell biological experiments using the SH-SY5Y control, ATP13A2 $\mathrm{KO}, \mathrm{KO} / \mathrm{WT}$ and $\mathrm{KO} / \mathrm{D} 508 \mathrm{~N}$ cell lines, each cell model is the sum of two independent clones, each performed a minimum of three independent times. For the quantification of immunoblots and radiograms, ImageJ and ImageQuant programmes were used. Experiments on various model systems were executed by different researchers, which provided consistent results that independently confirmed the major conclusions.

\section{Reporting summary}

Further information on research design is available in the Nature Research Reporting Summary linked to this paper.

\section{Data availability}

Gel source data for immunoblots and radiograms (Figs. 1, 2, Extended Data Figs.1-3,6) are available with the online version of the paper (Supplementary Fig. 1). All other datasets generated within this study are presented and analysed within this manuscript and are available from the corresponding author upon reasonable request.

31. Axelsen, K. B. \& Palmgren, M. G. Evolution of substrate specificities in the P-type ATPase superfamily. J. Mol. Evol. 46, 84-101 (1998).

32. Grunewald, A. et al. ATP13A2 mutations impair mitochondrial function in fibroblasts from patients with Kufor-Rakeb syndrome. Neurobiol Aging 33, 1843.e1-1843.e7 (2012)

33. Usenovic, M., Tresse, E., Mazzulli, J. R., Taylor, J. P. \& Krainc, D. Deficiency of ATP13A2 leads to lysosomal dysfunction, a-synuclein accumulation, and neurotoxicity. J. Neurosci. 32 4240-4246 (2012)

34. Santoro, L. et al. Novel ATP13A2 (PARK9) homozygous mutation in a family with marked phenotype variability. Neurogenetics 12, 33-39 (2011)

35. Funayama, M. et al. Rapid screening of ATP13A2 variant with high-resolution melting analysis. Mov. Disord. 25, 2434-2437 (2010).

36. Lin, C. H. et al. Novel ATP13A2 variant associated with Parkinson disease in Taiwan and Singapore. Neurology 71, 1727-1732 (2008).

37. Mao, X. Y. et al. ATP13A2 G2236A variant is rare in patients with early-onset Parkinson's disease and familial Parkinson's disease from mainland China. Parkinsonism Relat. Disord. 16, 235-236 (2010).

38. Sanjana, N. E., Shalem, O. \& Zhang, F. Improved vectors and genome-wide libraries for CRISPR screening. Nat. Methods 11, 783-784 (2014).

39. Kleinstiver, B. P. et al. High-fidelity CRISPR-Cas9 nucleases with no detectable genomewide off-target effects. Nature 529, 490-495 (2016).

40. Doench, J. G. et al. Optimized sgRNA design to maximize activity and minimize off-target effects of CRISPR-Cas9. Nat. Biotechnol. 34, 184-191 (2016).

41. Covy, J. P., Waxman, E. A. \& Giasson, B. I. Characterization of cellular protective effects of ATP13A2/PARK9 expression and alterations resulting from pathogenic mutants. J. Neurosci. Res. 90, 2306-2316 (2012).

42. Gitler, A. D. et al. a-synuclein is part of a diverse and highly conserved interaction network that includes PARK9 and manganese toxicity. Nat. Genet. 41, 308-315 (2009).

43. Kong, S. M. et al. Parkinson's disease-linked human PARK9/ATP13A2 maintains zinc homeostasis and promotes a-synuclein externalization via exosomes. Hum. Mol. Genet. 23, 2816-2833 (2014)

44. Lambie, E. J., Tieu, P. J., Lebedeva, N., Church, D. L. \& Conradt, B. CATP-6, a C. elegans ortholog of ATP13A2 PARK9, positively regulates GEM-1, an SLC16A transporter. PLOS ONE 8, e77202 (2013)

45. Tsunemi, T. et al. Increased lysosomal exocytosis induced by lysosomal $\mathrm{Ca}^{2+}$ channel agonists protects human dopaminergic neurons from a-synuclein toxicity. J. Neurosci. 39, 5760-5772 (2019).
46. Gietz, R. D. \& Woods, R. A. Transformation of yeast by lithium acetate/single-stranded carrier DNA/polyethylene glycol method. Methods Enzymol. 350, 87-96 (2002).

47. Azouaoui, H. et al. A high-yield co-expression system for the purification of an intact Drs2p-Cdc50p lipid flippase complex, critically dependent on and stabilized by phosphatidylinositol-4-phosphate. PLoS ONE 9, e112176 (2014).

48. Jidenko, M., Lenoir, G., Fuentes, J. M., le Maire, M. \& Jaxel, C. Expression in yeast and purification of a membrane protein, SERCA1a, using a biotinylated acceptor domain. Protein Expr. Purif. 48, 32-42 (2006).

49. Papadopulos, A. et al. Flippase activity detected with unlabeled lipids by shape changes of giant unilamellar vesicles. J. Biol. Chem. 282, 15559-15568 (2007).

50. Byun, J. A. et al. Analysis of polyamines as carbamoyl derivatives in urine and serum by liquid chromatography-tandem mass spectrometry. Biomed. Chromatogr. 22, 73-80 (2008).

51. Stroikin, Y., Mild, H., Johansson, U., Roberg, K. \& Ollinger, K. Lysosome-targeted stress reveals increased stability of lipofuscin-containing lysosomes. Age 30, 31-42 (2008)

52. Nilsson, C., Kagedal, K., Johansson, U. \& Ollinger, K. Analysis of cytosolic and lysosomal pH in apoptotic cells by flow cytometry. Methods Cell. Sci. 25, 185-194 (2003).

53. Osório, L. et al. Viral vectors expressing a single microRNA-based short-hairpin RNA result in potent gene silencing in vitro and in vivo. J. Biotechnol. 169, 71-81 (2014).

54. Brenner, S. The genetics of Caenorhabditis elegans. Genetics 77, 71-94 (1974).

55. Casadaban, M. J., Martinez-Arias, A., Shapira, S. K. \& Chou, J. ß-galactosidase gene fusions for analyzing gene expression in Escherichia coli and yeast. Methods Enzymol. 100, 293-308 (1983).

56. The C. elegans Deletion Mutant Consortium. Large-scale screening for targeted knockouts in the Caenorhabditis elegans genome. G3 2, 1415-1425 (2012).

57. Mello, C. C., Kramer, J. M., Stinchcomb, D. \& Ambros, V. Efficient gene transfer in C. elegans: extrachromosomal maintenance and integration of transforming sequences. EMBO J. 10, 3959-3970 (1991).

58. Roy, A., Kucukural, A. \& Zhang, Y. I-TASSER: a unified platform for automated protein structure and function prediction. Nat. Protoc. 5, 725-738 (2010).

59. Zhang, Y. I-TASSER server for protein 3D structure prediction. BMC Bioinformatics $\mathbf{9}, 40$ (2008).

60. Omasits, U., Ahrens, C. H., Müller, S. \& Wollscheid, B. Protter: interactive protein feature visualization and integration with experimental proteomic data. Bioinformatics $\mathbf{3 0}$ 884-886 (2014)

Acknowledgements This work was funded by the Fonds Wetenschappelijk Onderzoek (FWO Research Foundation Flanders) (G094219N to PV G092714 and G080517N to V. Baekelandt SBO Neuro-TRAFFIC S006617N to V. Baekelandt, P.V., W.A. and J.V.S. and 1503117 N to S.M.), the KU Leuven (OT/13/091; LysoCaN C16/15/073 to P.V., J.V.S., W.A. and P.A.; OT/14/120 to V. Baekelandt) and the Queen Elisabeth Medical Foundation for Neurosciences (P.V. and V. Baekelandt). S.v.V. is an aspirant FWO research fellow (11Y7518N) and J.P.K. is the recipient of a strategic basic research doctoral grant of the FWO (1S18518N). J.L. received Lundbeck postdoctoral fellowships R209-2015-2704 and R171-2014-663. Funding for E.L. and J.Z. was provided by DFG grant LA3380/2-1. Some nematode strains were provided by the Caenorhabditis Genetics Center, which is funded by NIH Office of Research Infrastructure Programs (P40 OD010440). We thank P. P. Van Veldhoven (KU Leuven) for discussions; P. Chaltin (Center for Drug Design and Discovery) for financial support for V. Benoy; and M. Schuermans, T. Arslan, A. Florizoone, J. Van Asselberghs, J. Chen and N. Hamouda for technical assistance. We also acknowledge our frequent use of the facilities and equipment of the Leuven Viral Vector Core facility (KU Leuven), Mass Spectrometry Core (R. Derua, KU Leuven), Cell and Tissue Imaging Cluster (P. Vanden Berghe, KU Leuven) and the FACS Core (KU Leuven/VIB).

Author contributions The study was designed by P.V., S.M., S.v.V. and J.E.; C.V.d.H. generated all stable cell lines and analysed ATP13A2 knockdown. J.-P.D. performed and analysed the CRISPR experiments; R.V. and J.P.K. synthesized BODIPY-labelled polyamine analogues; J.P.K performed and analysed acidic nanoparticle preparations; S.v.V performed and analysed the biochemical experiments (except Extended Data Fig. $2 f$ by V. Benoy); J.L. contributed to the ATP13A2 purification protocol; S.M. performed and analysed the cell biology experiments; B.G. performed metabolomics; G.G. isolated mouse neurons; E.L. and J.Z. performed C. elegans experiments; and P.V., S.v.V. and S.M. wrote the manuscript, which was reviewed by all authors.

Competing interests Patent WO-2018002350-A1 of KU Leuven describes methods for detecting compounds with therapeutic use that target ATP13A2 or related isoforms using biological material and assays described in the current manuscript. A second patent of $K U$ Leuven describing ATP13A2 cell models described in this manuscript has also been filed.

Additional information

Supplementary information is available for this paper at https://doi.org/10.1038/s41586-020 1968-7.

Correspondence and requests for materials should be addressed to P.V.

Peer review information Nature thanks Guillaume Lenoir, Hanne Poulsen, Taiji Tsunemi and the other, anonymous, reviewer(s) for their contribution to the peer review of this work.

Reprints and permissions information is available at http://www.nature.com/reprints. 


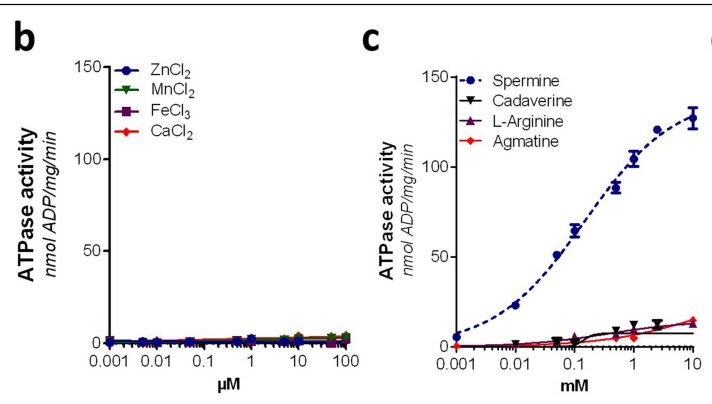

g
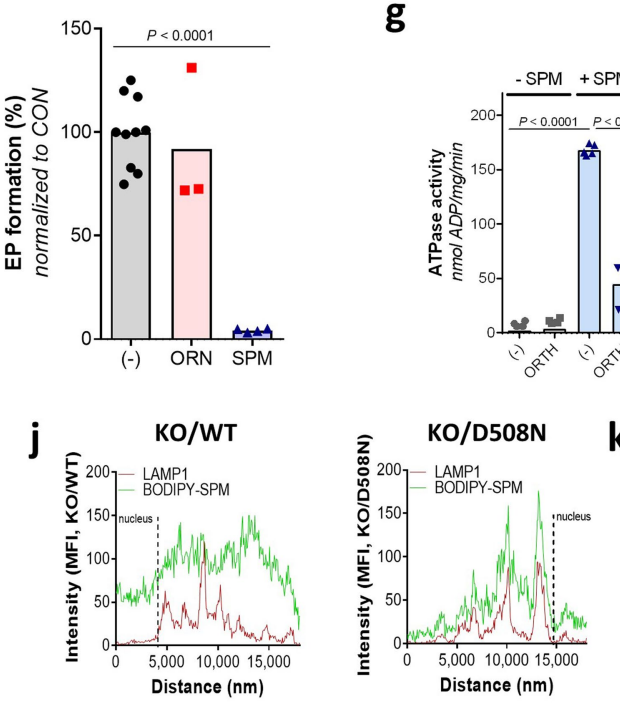
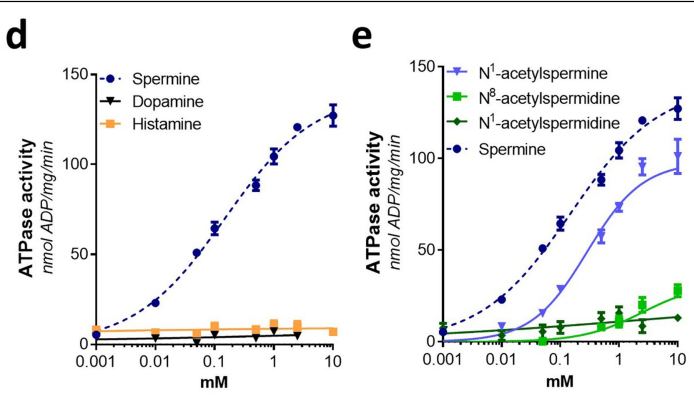

h
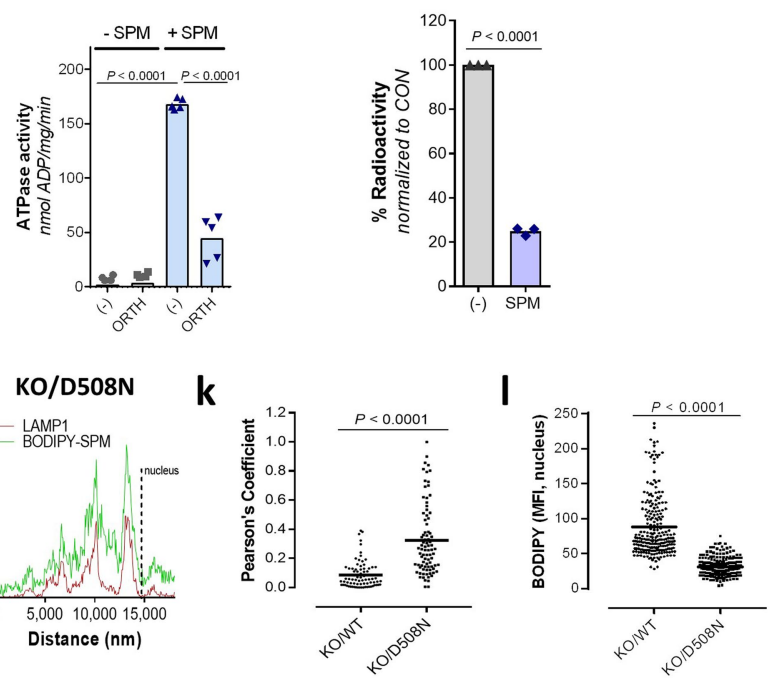

h, Purified ATP13A2 was incubated with $\left[\gamma^{-32} \mathrm{P}\right]$ ATP in the presence of $1 \mathrm{mMSPM}$, and radioactivity of the phospho-intermediate was assessed by scintillation counting. i, Comparison of the pulse $(5 \mu \mathrm{M}, 15 \mathrm{~min})$ chase (105 min, medium) BODIPY-SPM uptake in KO/WT and KO/D508N cell lines by confocal microscopy. Cells were subsequently stained with LAMP1 and imaged with the same laser settings by confocal microscopy. DAPI was used to visualize the nuclei. Scale bar, $5 \mu \mathrm{m}$.j, Line intensity plots of the indicated dashed lines in Fig. 2d.k, Analysis of the Pearson's coefficient of LAMP1 and BODIPY-SPM for the images in Fig. 2d (KO/WT, 78 images; KO/D508N, 85 images). I, Mean fluorescence intensities (MFI) of BODIPY in DAPI-positive regions of samples shown in Fig. 2d (KO/WT, 233 nuclei; KO/D508N, 243 nuclei). Data are presented as the mean \pm s.e.m. or mean with individual data points shown (points represent replicates), with $n=3$ independent biological experiments. Analysis was carried out using one-way ANOVA with Dunnett's (f) or Tukey's (a, g) corrections, or by two-tailed $t$-tests (unpaired, hor Welch's, k, I). Fitted lines are semi-log lines (b) or nonlinear allosteric sigmoidal association (c-e). For gel source data, see Supplementary Fig. 1. 

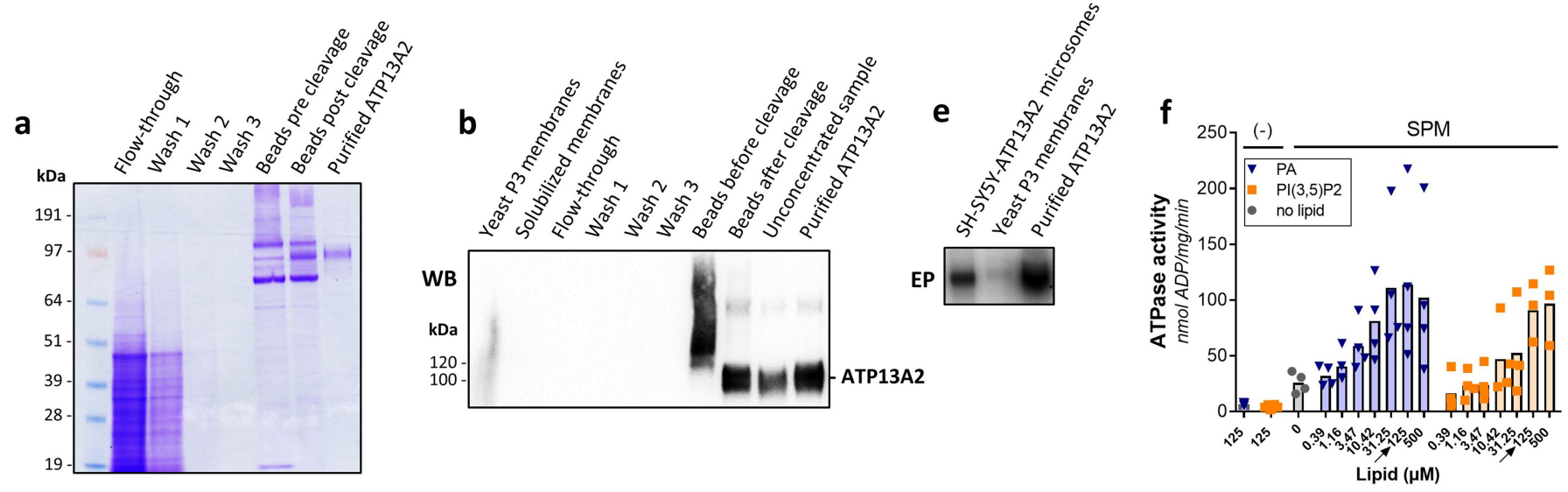

c

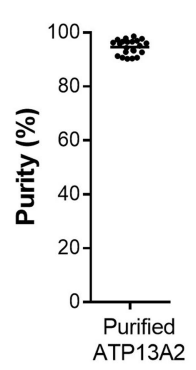

d

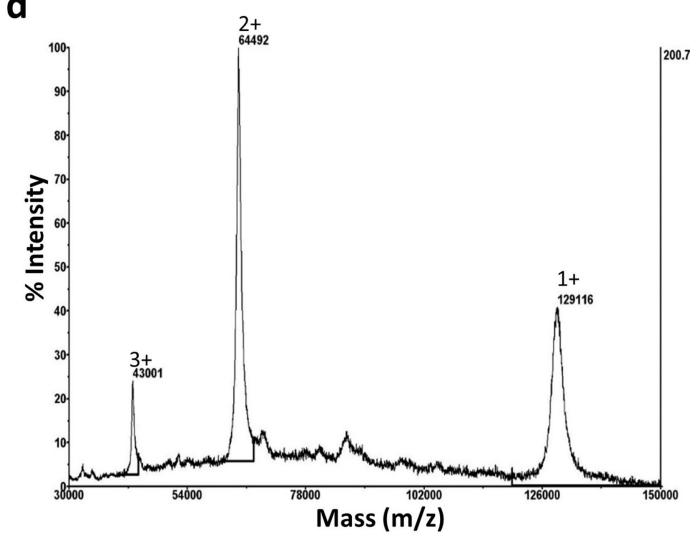

g

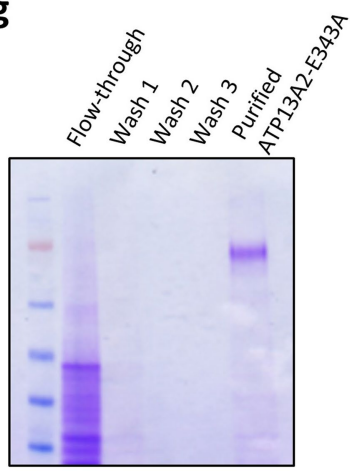

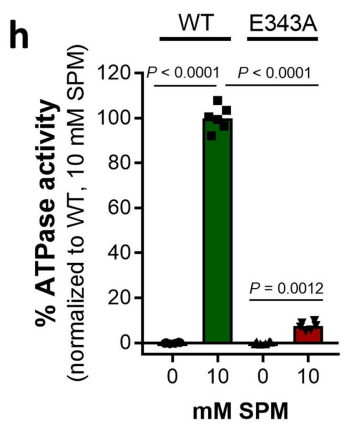

Extended Data Fig. 2 | Streptavidin-based purification of wild-type ATP13A2 and the catalytically dead E343A mutant. a, Coomassie staining showing the purification process for wild-type ATP13A2, starting from solubilized yeast membrane fractions, followed by streptavidin affinity chromatography and on-column thrombin cleavage to elute the protein. b. Western blot analysis of stages in the purification of ATP13A2.c, Bar graph depicting protein purity as determined by densitometry from Coomassie-stained SDS-PAGE.d, Mass spectrometry analysis of the purified ATP13A2 sample. Singly, doubly and triply charged species are indicated.e, To evaluate phosphoenzyme formation, yeast P3 membranes $(20 \mu \mathrm{g})$ and purified ATP13A2 $(1 \mu \mathrm{g})$ were incubated for $60 \mathrm{~s}$ with [ $\left.\mathrm{\gamma}^{-32} \mathrm{P}\right] \mathrm{ATP}$. As a positive control, microsomes collected from SH-SY5Y cells that overexpress wild-type ATP13A2 $(20 \mu \mathrm{g})$ were used. The image is a representative radiogram depicting the ATP13A2 phosphoenzyme.f, The ATPase activity of purified ATP13A2 $(0.3 \mu \mathrm{g})$ was measured in the presence of $2 \mathrm{mMSPM}$ and the indicated concentrations of the ATP13A2 regulatory lipids phosphatidic acid (PA) and PtdIns $(3,5) \mathrm{P}_{2}$. g, Coomassie staining showing the purification process for ATP13A2(E343A). $\mathbf{h}$, The ATPase activity of purified wild-type ATP13A2 or ATP13A2(E343A) $(0.5 \mu \mathrm{g})$ was measured in the presence of the indicated concentrations of SPM with $125 \mu \mathrm{M}$ phosphatidic acid and $125 \mu \mathrm{MPtd} \operatorname{lns}(3,5) \mathrm{P}_{2}$. Data are expressed as mean with individual data points (points represent replicates) (c, h). The number of independent biological experiments were as follows: $n=3(\mathbf{b}, \mathbf{e}-\mathbf{h}) ; n=6(\mathbf{d}) ; n=22(\mathbf{a}, \mathbf{c})$. Analysis was performed using one-way ANOVA with Tukey's post-hoc correction (h). For gel source data, see Supplementary Fig. 1. 
a

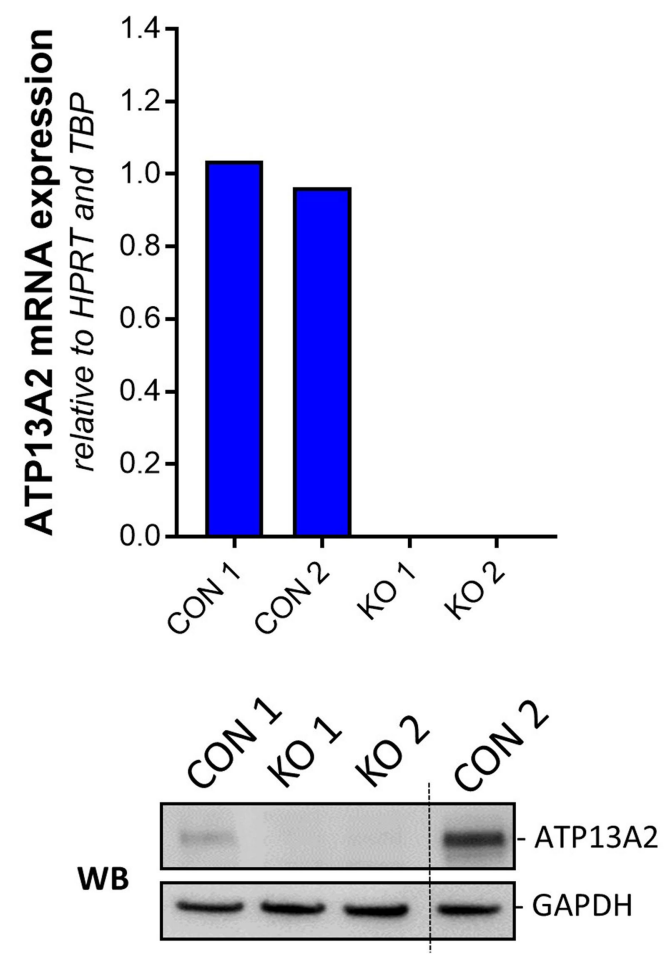

Extended Data Fig. 3 | Confirmation of CRISPR-Cas9-mediated ATP13A2 knockout and subsequent rescue with wild-type ATP13A2 or the D508N

mutant. a, The ATP13A2 knockout cell lines (KO) were generated by CRISPRCas9 in SH-SY5Y cells and confirmed by qPCR (top) and immunoblotting (bottom).Atp13a2 mRNA expression was normalized to hypoxanthine phosphoribosyltransferase (HPRT) and TATA-sequence-binding protein (TBP), and GAPDH was used as a loading control for the ATP13A2 protein levels. Two



WB

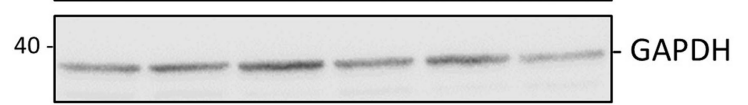

fragments of the same blot are depicted and separated by a dotted line. b, Rescue of ATP13A2 knockout was performed by lentiviral transduction to express wild-type ATP13A2 (KO/WT) and the catalytically dead mutant D508N, which was used as a negative control (KO/D508N). The expression of the ATP13A2 constructs was confirmed via immunoblotting. The number of biologically independent experiments were as follows: $n=1$ (a, top panel); $n=3$ (a, bottom panel, b). For gel source data, see Supplementary Fig.1. 
a

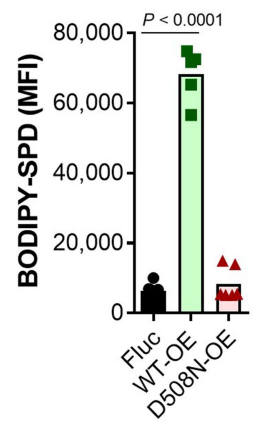

b

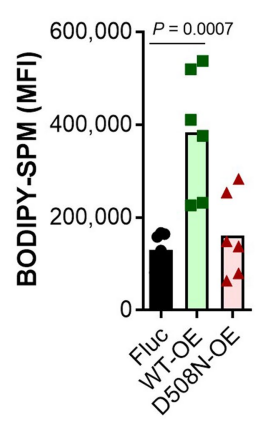

d



$\mathbf{f}$

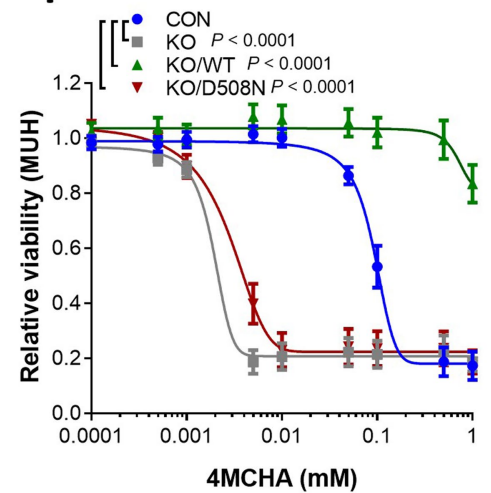

e

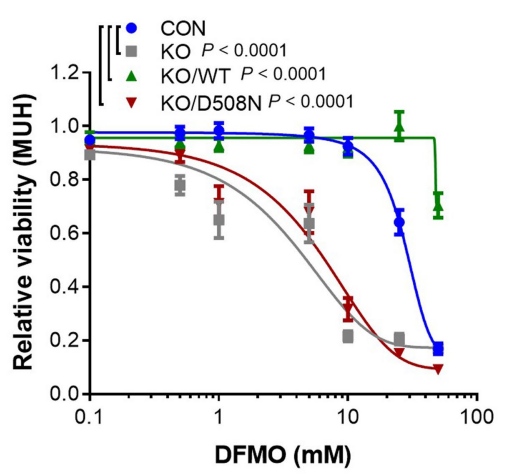

g

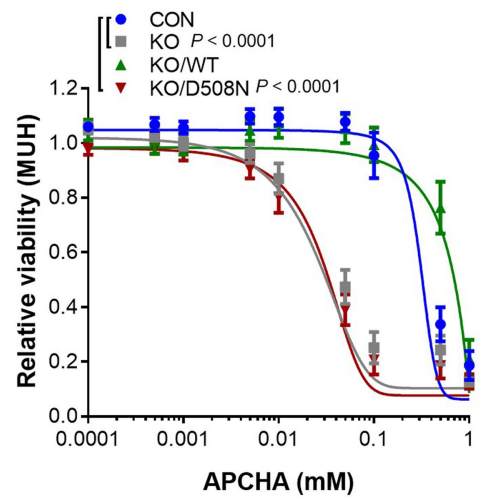

Extended Data Fig. 4 | Polyamine uptake by ATP13A2 complements cytosolic polyamine synthesis. a-c, Assessment of the cellular uptake of BODIPYlabelled polyamine analogues $(\mathbf{a}, \mathbf{b})$ or FITC-dextran (c) by flow cytometry. Uptake of BODIPY-SPD (a) or BODIPY-SPM (b) in SH-SY5Y cells overexpressing Fluc (negative control), wild-type ATP13A2 (WT-OE) or the catalytically dead mutant D508N (D508N-OE). The cells were incubated with $5 \mu$ M BODIPY-SPM or BODIPY-SPD for $2 \mathrm{~h}$ before analysis by flow cytometry. $\mathrm{c}$, Analysis of FITCdextran uptake (as a measure of endocytic capacity) was performed in SH-SY5Y control (CON) cells with endogenous ATP13A2 expression, ATP13A2 knockout cells (KO) and rescue cell lines with expression of wild-type ATP13A2 (KO/WT) or the D508N mutant (KO/D508N). The cells were pre-treated for $30 \mathrm{~min}$ with a combination (combo) of endocytosis inhibitors Dynasore $(100 \mu \mathrm{M})$, genistein $(50 \mu \mathrm{M})$ and Pitstop $2(50 \mu \mathrm{M})$. The cells were incubated for an additional $2 \mathrm{~h}$ with FITC-dextran at $37^{\circ} \mathrm{C}$, followed by flow cytometry. d, Schematic representation of polyamine synthesis. SRM, spermidine synthase; SMS, spermine synthase. Specific inhibitors are indicated in red. Control, $\mathrm{KO}, \mathrm{KO} /$ WT or KO/D508N cells were subjected to inhibition of polyamine synthesis by $\operatorname{DFMO}(\mathbf{e}), 4 \mathrm{MCHA}(\mathbf{f})$ and APCHA $(\mathbf{g})$ before measuring cell viability via the MUH assay. All data represent the average of two independent CRISPR-Cas 9 knockout and control clones. All data are presented as mean with data points overlaid (points represent replicates) or mean \pm s.e.m. The number of biologically independent experiments were as follows: $n=3(\mathbf{a}, \mathbf{b}) ; n=4(\mathbf{c}, \mathbf{e}-\mathbf{g})$. Analysis was performed using one-way ANOVA with Dunnett's $(\mathbf{a}, \mathbf{b})$ or Tukey's (c) post-hoc correction or two-way ANOVA with Dunnett's post-hoc correction (e-g). Fitted lines indicate nonlinear $\log$ (inhibitor) versus response (variable slope) (e-g). 
a



b



e

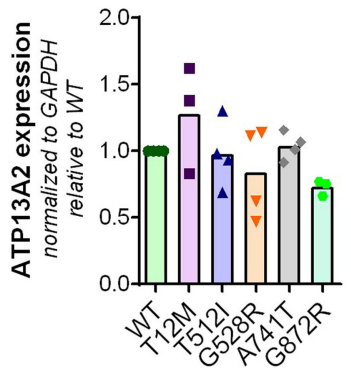

Extended Data Fig. 5 | Catalytic and clinical mutations of ATP13A2 perturb polyamine function. a, Sequence alignment of predicted transmembrane helices M4 (left), M6 (middle) and M8 (right). The alignment was generated using Clustal Omega. We generated mutants in M4 (A467V), M6 (D962N) and M8 (K1062A). The A467V mutation converts the protein sequence PPALP of the predicted substrate-binding site in transmembrane segment $M 4$ into the protein sequence PPVLP that is present in ATP13A $5^{4,30}$. Neighbouring membrane helices also contribute to substrate coordination in P-type ATPases, which often relies on conserved and charged residues, such as D962 in M6 and K1062 in M8 of ATP13A2 ${ }^{4}$.b, Densitometry of the expression of catalytic mutants presented in Fig. 2e. c, Flow cytometric analysis of cellular BODIPYSPD uptake in SH-SY5Y cells overexpressing wild-type ATP13A2, the D508N mutant or catalytic mutants. d, Quantification of ATP13A2 phosphorylation levels (EP) presented in Fig. 2g. e, Densitometry analysis of the expression of



C

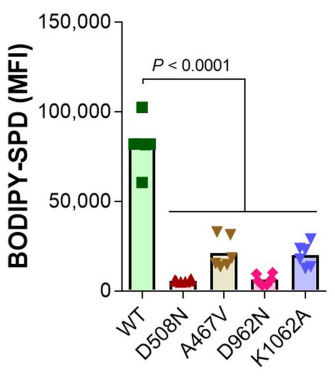

d



g

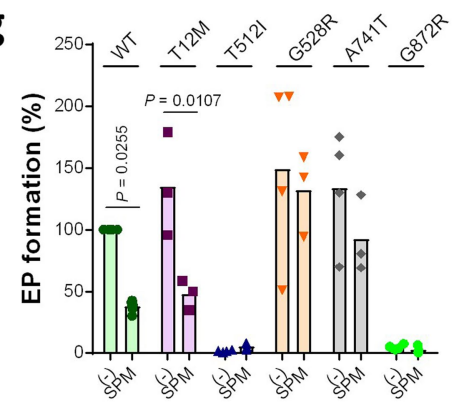

disease-related mutants presented in Fig. 2i.f, Flow cytometric analysis of cellular BODIPY-SPD uptake in SH-SY5Y cells overexpressing wild-type ATP13A2, D508N or disease mutants. g, Quantification of ATP13A2 phosphorylation levels presented in Fig. $2 \mathrm{k}$. All data are depicted as mean with individual data points (points represent replicates). The number of independent biological experiments were as follows: $n=3 \mathbf{b}, \mathbf{c}, \mathbf{d}$ (D508N (SPM), A467V (SPM), D962N (SPM), and K1062A (SPM)), e (T12M, G872R), f, g (T12M (-), T12M (SPM), T512I (SPM), G528R (SPM), A741T (SPM), and G872R (SPM)); $n=4$ d (D508N (-), A467V (-), D962N (-), and K1062A (-)), e (wild-type, T512I, G528R and A741T), $\mathbf{g}$ (wild-type (-), T512I (-), G528R (-), A741T (-), and G872R $(-)$ ); $n=5 \mathbf{d}$ (wild-type (-)); $n=6 \mathbf{d}$ (wild-type (SPM)) and $\mathbf{g}$ (wild-type (SPM)). Analysis by one-way ANOVA with Dunnett's (b, c, e,f) or two-way ANOVA with Sidak's $(\mathbf{d}, \mathbf{g})$ post-hoc corrections. 


\begin{tabular}{cccc}
\hline & Source & $\begin{array}{c}\text { K } \pm \text { SEM }\left(s^{-1}\right) \\
\text { ATP sensitivity }\end{array}$ & $\begin{array}{c}\text { K } \pm \text { SEM }\left(s^{-1}\right) \\
\text { ATP/SPM sensitivity }\end{array}$ \\
\hline ATP13A2 WT & Microsomes & $0.066 \pm 0.010$ & $0.098 \pm 0.008$ \\
\hline ATP13A2 D962N & Microsomes & $0.022 \pm 0.019$ & $0.020 \pm 0.012$ \\
\hline ATP13A2 WT & Purified protein & $0.050 \pm 0.012$ & $0.136 \pm 0.030$ \\
\hline ATP13A2 E343A & Purified protein & $0.035 \pm 0.031$ & $0.039 \pm 0.015$ \\
\hline
\end{tabular}

\begin{tabular}{|c|c|}
\hline \multicolumn{2}{|c|}{ Time after addition (s) } \\
\hline cold ATP & cold ATP/SPM \\
\hline
\end{tabular}

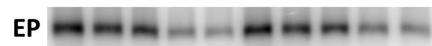

ATP13A2 D962N

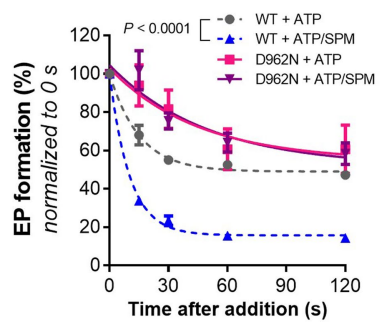

Extended Data Fig. 6 | The ATP sensitivity of ATP13A2(D962N) and ATP13A2(E343A) is independent of SPM. a, Overview of rate constants of ATP13A2 phosphoenzyme decay following a chase with non-radioactive ATP with or without $1 \mathrm{mM} \mathrm{SPM}$. b, After $30 \mathrm{~s}$ of incubating D962N microsomes (20 $\mu \mathrm{g})$ with $\left[\mathrm{\gamma}^{-32} \mathrm{P}\right] \mathrm{ATP}$, the time course of dephosphorylation after an ATP chase was measured in the presence or absence of SPM. The top panel shows a representative autoradiogram of the phosphoenzymes (EP), whereas the bottom panel depicts the quantification of ATP13A2 phosphorylation levels. As a reference we plotted the wild-type curve, shown in Fig.1d. Data are presented as the mean \pm s.e.m. of $n=4$ biologically independent experiments. Analysis by two-way ANOVA with Tukey's test (b). The fitted line indicates two-phase decay (b). For gel source data, see Supplementary Fig. 1. 
a

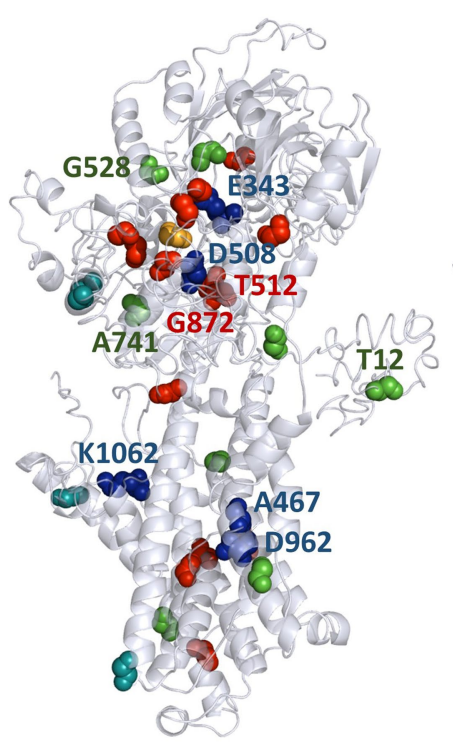

b

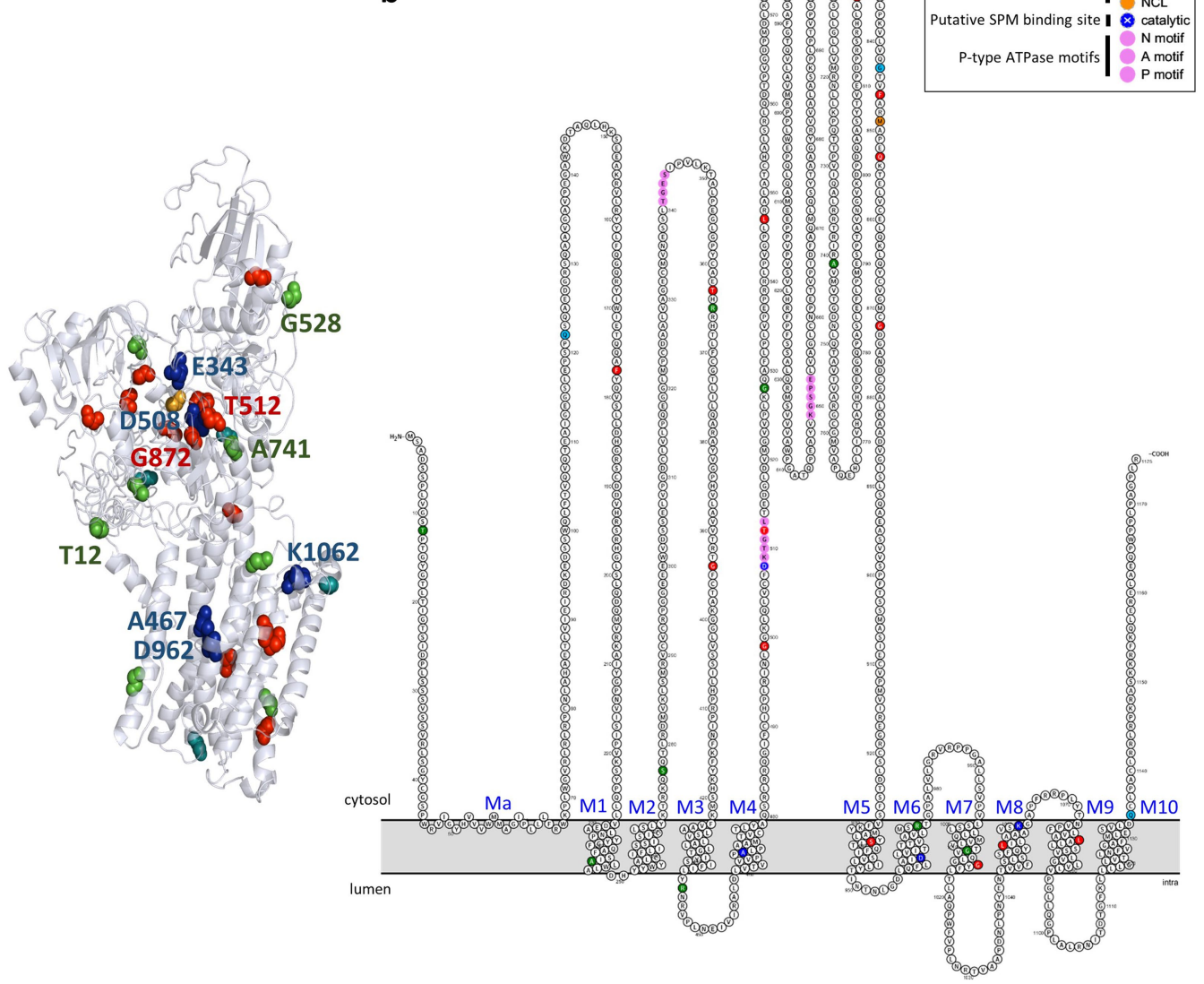

onset Parkinson's disease (PD)-associated mutations in green; hereditary spastic paraplegia (HSP)-associated mutations in light blue; neuronal ceroid lipofuscinosis (NCL)-associated mutations in orange. Catalytic mutations and mutations in the predicted substrate-binding region are highlighted in dark blue. Residues that were subjected to mutagenesis in this study are labelled (only in a). P-type ATPase signature motifs in the cytosolic domains are indicated in pink (only in b). helices (M1-10) and an N-terminal membrane-associated region (Ma) ${ }^{9}$. KuforRakeb syndrome (KRS)-associated mutations are highlighted in red; early-
Extended Data Fig. 7 |Predicted topology of ATP13A2.a, Homology model of ATP13A2 based on the structure of $\mathrm{Na}^{+} / \mathrm{K}^{+}$-ATPase (ATP1A1, PDB ID:3A3Y) as a template, generated by iTASSER (https://zhanglab.ccmb.med.umich.edu/lTASSER $/)^{58,59} \cdot \mathbf{b}$, Predicted membrane topology of ATP13A 2 visualized by 

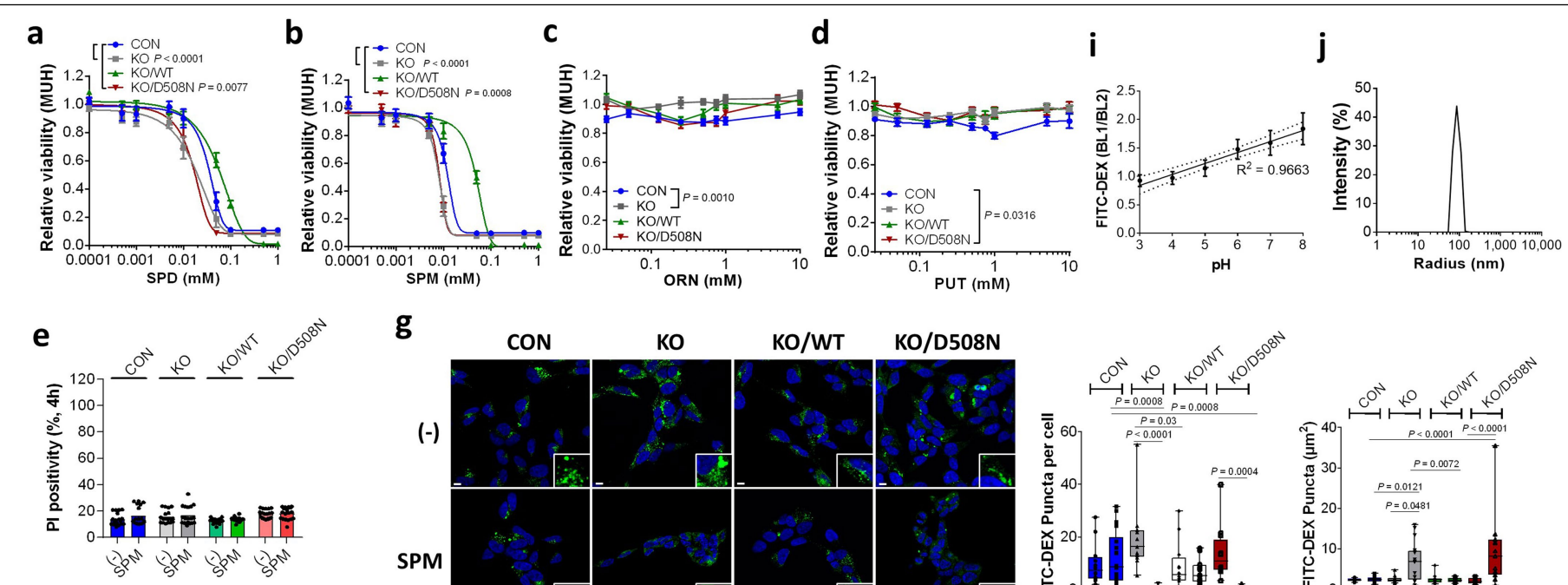

g CON
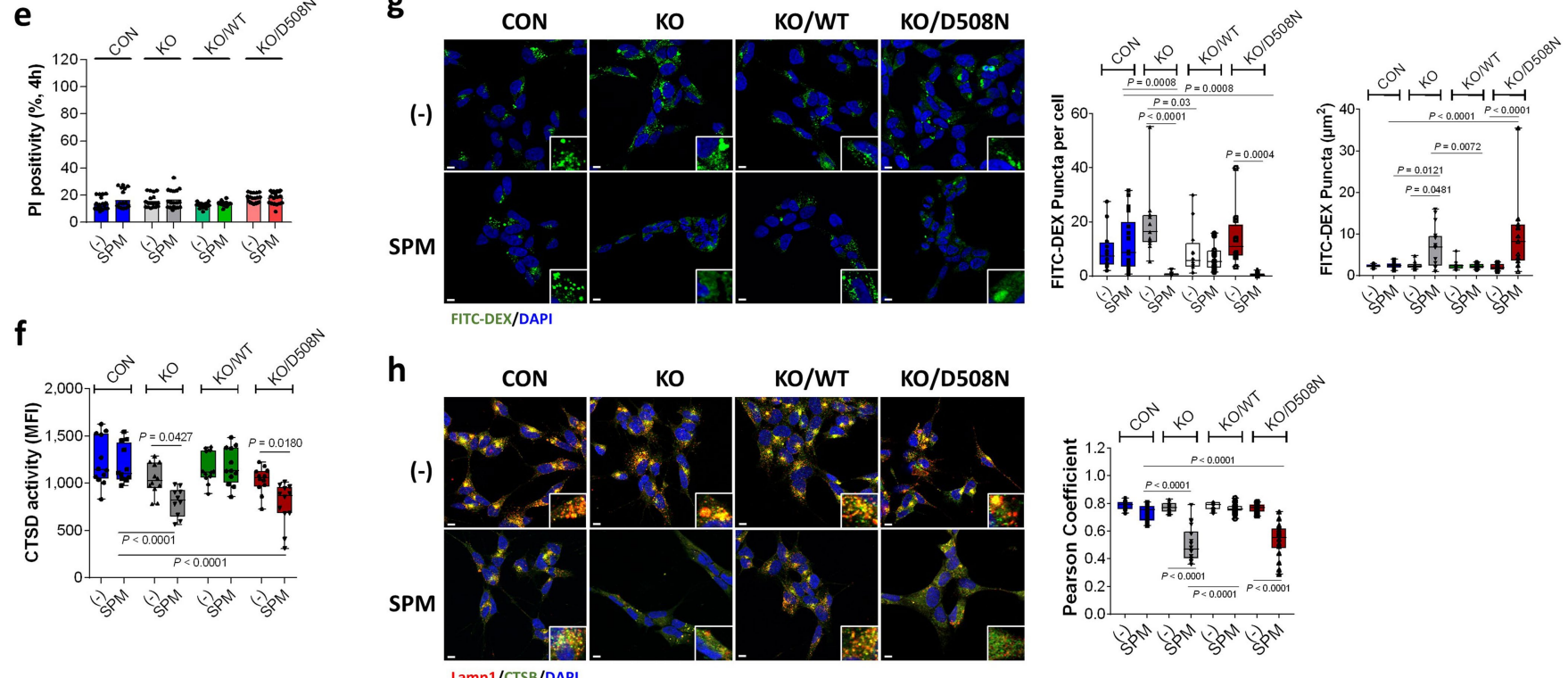

Extended Data Fig. 8 | Lysosomal functionality and recovery. a-d, The impact of exogenous polyamines on cell toxicity $(24 \mathrm{~h})$ and lysosomal functionality $(4 \mathrm{~h})$ was assessed in $\mathrm{SH}-\mathrm{SY} 5 \mathrm{Y}$ control cells (CON) with endogenous ATP13A2, ATP13A2 knockout cells (KO) and rescue cell lines with wild-type expression (KO/WT) or expression of the catalytically dead mutant D508N (KO/D508N) on the KO background. Cytotoxicity of SPD (a), SPM (b), ORN (c) and PUT (d) were assessed via a MUH cell-viability assay. e, Death of the aforementioned cells was assessed after $4 \mathrm{~h}$ of SPM exposure $(10 \mu \mathrm{M})$ by propidium iodide (PI)-based flow cytometry. $\mathbf{f}$, Measurement of cathepsin D activity. $\mathbf{g}, \mathbf{h}$, Lysosomal rupture under basal (-) and SPM $(10 \mu \mathrm{M})$ conditions was assessed via loss of FITC-dextran (FITC-DEX) punctae (g) or loss of cathepsin B (CTSB)/LAMP1 colocalization (h). Confocal images depict representative images with or without SPM exposure ( $4 \mathrm{~h}$, DAPI staining for nuclei was included as a reference). Scale bars, $10 \mu \mathrm{m}$. The box and whisker plots in $\mathbf{g}$ depict the frequency (left) and size (right) of FITC-DEX punctae; in $\mathbf{h}$

the Pearson coefficient of colocalization of cathepsin B and LAMP1. i, Lysosomal pH (Fig. 3b) was evaluated using the fluorescent probe FITCdextran and a dual-emission ratiometric technique. FITC is excited at $488 \mathrm{~nm}$ and emission is analysed at $530 \mathrm{~nm}$ (BL1) and $610 \mathrm{~nm}$ (BL2). A pH calibration curve was generated using FITC-dextran in cells permeabilized with $100 \mu \mathrm{M}$ monensin and equilibrated with calibration buffers ( $\mathrm{pH} 3-8)$.j, Representative size distribution of the acidic nanoparticles used in this study. Data are presented as the mean \pm s.e.m. (a-d, $\mathbf{i}$ ) or individual data points (representing replicates) overlaid on group means (e) or box and whisker plots (f-h, line, median; box boundaries, 25 th and 75 th percentiles). The number of independent biological experiments were as follows: $n=3(\mathbf{e}-\mathbf{j}) ; n=4(\mathbf{a}-\mathbf{d})$. Analysis was performed using two-way ANOVA with Dunnett's $(\mathbf{a}, \mathbf{b})$ or Bonferroni's (c, d) post-hoc corrections, or one-way ANOVA with Dunnett's (e), Sidak's (f, $\mathbf{g}$ (right)) or Tukey's ( $\mathbf{g}$ (left), h) post-hoc corrections. Fitted lines indicate nonlinear $\log$ (inhibitor) versus response (variable slope) $(\mathbf{a}, \mathbf{b})$. 


\section{Article}
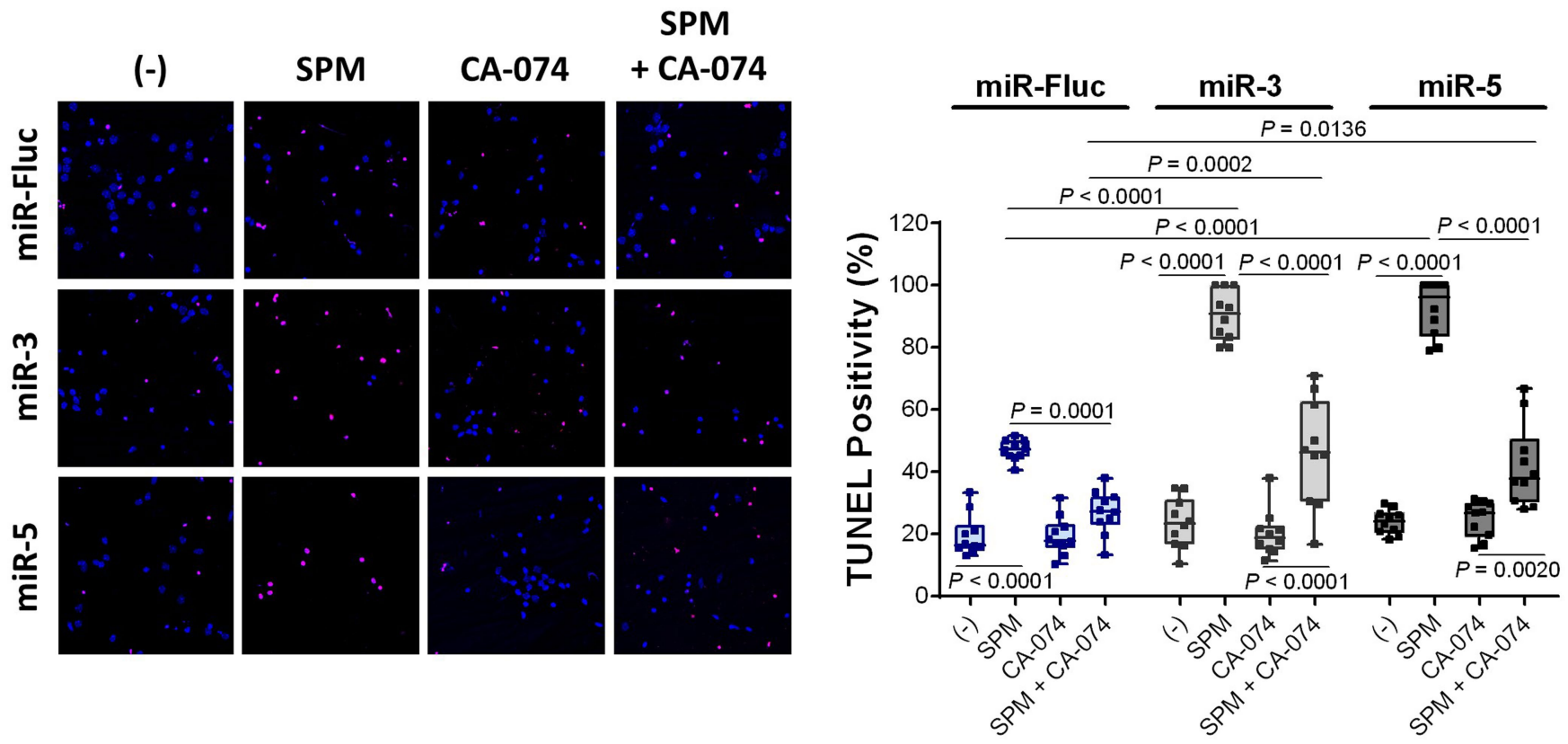

Extended Data Fig. 9 | Inhibition of cathepsin B activity attenuates SPMinduced neuronal death. The effect of a cathepsin B inhibitor (CA-074, 10 $\mu \mathrm{M})$ on SPM-induced $(10 \mu \mathrm{M}, 24 \mathrm{~h})$ cell death in control (miR-Fluc) and Atp13a2

box and whisker plots with the quantification of the TUNEL staining. Data are presented as box and whisker plots (line, median; box boundaries, 25th and knockdown (miR-3 and miR-5) neurons was assayed via TUNEL-based staining. Left, representative confocal images depicting TUNEL-positive neurons; right, 75th percentiles) for which individual data points (representing replicates) are shown. $n=3$ biologically independent experiments. Analysis by one-way ANOVA with Tukey's post-hoc correction. 
Extended Data Table 1 | Apparent $K_{\mathrm{m}}$ and $V_{\max }$ values for ATP13A2 in the presence of various polyamines

\begin{tabular}{|c|c|c|c|c|}
\hline & \multicolumn{2}{|c|}{$\mathrm{K}_{\mathrm{m}} \pm \mathrm{SEM}(\mu \mathrm{M})$} & \multicolumn{2}{|c|}{$\mathrm{V}_{\max } \pm \mathrm{SEM}$ (nmol ADP/mg/min) } \\
\hline & Microsomal ATP13A2 & Purified ATP13A2 & Microsomal ATP13A2 & Purified ATP13A2 \\
\hline Spermine & $149 \pm 34$ & $76 \pm 26$ & $140 \pm 6$ & $159 \pm 10$ \\
\hline $\mathrm{N}^{1}$-acetylspermine & $286 \pm 48$ & not tested & $98 \pm 5$ & not tested \\
\hline Spermidine & 1700* & $890 \pm 501$ & 106* & $194 \pm 26$ \\
\hline
\end{tabular}

Data are derived from Fig. 1b, f and Extended Data Fig. 1e. Data represent $n=3$ (spermidine, $N^{\prime}$-acetylspermine), or $n=6$ (spermine) biologically independent experiments. * Estimated values (could not be accurately determined following fitting of the curve). 


\section{natureresearch}

Corresponding author(s): Peter Vangheluwe

Last updated by author(s): 21.11 .2019

\section{Reporting Summary}

Nature Research wishes to improve the reproducibility of the work that we publish. This form provides structure for consistency and transparency in reporting. For further information on Nature Research policies, see Authors \& Referees and the Editorial Policy Checklist.

\section{Statistics}

For all statistical analyses, confirm that the following items are present in the figure legend, table legend, main text, or Methods section.

n/a Confirmed

$\square$ \The exact sample size $(n)$ for each experimental group/condition, given as a discrete number and unit of measurement

Х $\square$ A statement on whether measurements were taken from distinct samples or whether the same sample was measured repeatedly

$\square$ The statistical test(s) used AND whether they are one- or two-sided

Only common tests should be described solely by name; describe more complex techniques in the Methods section.

$\square$ \ A description of all covariates tested

$\square$ \ A description of any assumptions or corrections, such as tests of normality and adjustment for multiple comparisons

$\square$ A full description of the statistical parameters including central tendency (e.g. means) or other basic estimates (e.g. regression coefficient)

$\triangle$ AND variation (e.g. standard deviation) or associated estimates of uncertainty (e.g. confidence intervals)

$\square$ For null hypothesis testing, the test statistic (e.g. $F, t, r$ ) with confidence intervals, effect sizes, degrees of freedom and $P$ value noted

$\square$ Give $P$ values as exact values whenever suitable.

Х $\square$ For Bayesian analysis, information on the choice of priors and Markov chain Monte Carlo settings

Х $\square$ For hierarchical and complex designs, identification of the appropriate level for tests and full reporting of outcomes

Х $\square$ Estimates of effect sizes (e.g. Cohen's $d$, Pearson's $r$ ), indicating how they were calculated

Our web collection on statistics for biologists contains articles on many of the points above.

\section{Software and code}

Policy information about availability of computer code

Data collection Typhoon TM FLA 9500 (phosphorimaging)

Flexstation 3 microplate reader (fluorescent and luminescent assays)

Bio-Rad ChemiDoc (western blot imager)

QuantaSmart TM (v2.03) (scintillation counting)

Attune Cytometric Software (v2.1) (flow cytometry)

ZEN SP5 2012 (confocal imaging)

Data analysis

GraphPad Prism Version 7.04 was used for data representation and statistical analysis. Image J and Zen 2.3 Lite were used for image analysis. Image Quant TL (version 8.1) was used for analysis of gel-based auto-phosphorylation assays. Image Lab (5.2.1) was used to analyze Western blots. XCalibur Quan tool (version 4.2.28.14) was used for polyamine quantification (LC MS). Flowing Software (v2.5.1) was used for flow cytometry analysis. i-TASSER (Protein struture and function predictions, https://zhanglab.ccmb. med.umich.edu/ITASSER/). PYMOL (structure visualization, version 2.3)

For manuscripts utilizing custom algorithms or software that are central to the research but not yet described in published literature, software must be made available to editors/reviewers. We strongly encourage code deposition in a community repository (e.g. GitHub). See the Nature Research guidelines for submitting code \& software for further information.

\section{Data}

Policy information about availability of data

All manuscripts must include a data availability statement. This statement should provide the following information, where applicable:

- Accession codes, unique identifiers, or web links for publicly available datasets

- A list of figures that have associated raw data

- A description of any restrictions on data availability

Source data for immunoblots and radiograms (Fig. 1-2, Extended data Fig. 1-3, 6) are available with the online version of the paper. All other datasets generated 


\section{Field-specific reporting}

Please select the one below that is the best fit for your research. If you are not sure, read the appropriate sections before making your selection. \ Life sciences $\quad \square$ Behavioural \& social sciences $\square$ Ecological, evolutionary \& environmental sciences

For a reference copy of the document with all sections, see nature.com/documents/nr-reporting-summary-flat.pdf

\section{Life sciences study design}

All studies must disclose on these points even when the disclosure is negative.

Sample size No statistical methods were used to pre-determine sample size. All fundamental experiments were performed a minimum of three times (independent biological experiments), including all relevant controls, to allow for the generation of S.E.M. Confirmation of ATP13A2 knockout (SH-SY5Y) was performed only once by qRT-PCR and N = 3 by western blot analysis. Knockdown of ATP13A2 in neurons was only confirmed two times by qRT-PCR.

Data exclusions No data were excluded.

Replication All experimental findings were reproduced in several independent biological experiments ( $N$ ) with multiple technical replicates. For each figure panel, the number of independent experiments $\mathrm{N}$ is indicated in the figure legends. Conclusions were independently confirmed in different model systems (in vitro, in cell lines, isolated neurons and in vivo) handled by multiple researchers and across several laboratories.

Randomization Samples were not randomized, but appropriate controls were included in each figure. For in vivo studies (nematodes), animals were evenly distributed such that each group had a similar mean/density at the start of the study.

Blinding Analyses were not blinded because experiments were performed and analyzed by the same researchers. Biochemistry, cell biology and in vivo research were performed independently by different researchers and their findings support one another providing independent confirmation.

\section{Reporting for specific materials, systems and methods}

We require information from authors about some types of materials, experimental systems and methods used in many studies. Here, indicate whether each material, system or method listed is relevant to your study. If you are not sure if a list item applies to your research, read the appropriate section before selecting a response.

Materials \& experimental systems

\begin{tabular}{l|l}
\hline Ia & Involved in the study \\
$\square$ Antibodies \\
$\square$ Eukaryotic cell lines \\
$\square$ Palaeontology \\
$\square$ Clinical data
\end{tabular}

\author{
Methods \\ $\mathrm{n} / \mathrm{a}$ Involved in the study \\ $\bigotimes \square$ ChIP-seq \\ $\square$ \ Flow cytometry \\ \ $\square$ MRI-based neuroimaging
}

\section{Antibodies}

Antibodies used

Validation
Primary antibodies used in this study with supplier, catalog number and lot number, as well as the used dilution.

SIGMA

anti-ATP13A2 antibody; catalogue number A3361, lot \# 076M4839, (Western Blotting, Rabbit, 1:1000) anti-GAPDH antibody; catalogue number G8795, lot \# 076M4785V, (Western Blotting, Mouse, 1:5000)

ABCAM

anti-galectin-3 antibody; catalogue number ab2785, lot \# GR3200865-11, (Immunofluorescence, Mouse, 1:200) anti-cathepsin B antibody; catalogue number ab58802, lot \# GR3181508-13, (Immunofluorescence, Mouse, 1:100) anti-lamp1 antibody; catalogue number ab24170, lot \# GR3235359-1, (Immunofluorescence, Rabbit, 1:200)

Concerning antibody specificity, we kindly refer to the supplier's websites and datasheets to find statements on specificity and citations for the use of the antibodies:

anti-ATP13A2 antibody; https://www.sigmaaldrich.com/catalog/product/sigma/a3361?lang=en\&region=BE anti-GAPDH antibody; https://www.sigmaaldrich.com/catalog/product/sigma/g8795?lang=en\&region=BE anti-galectin-3 antibody; https://www.abcam.com/galectin-3-antibody-a3a12-ab2785.html 


\section{Eukaryotic cell lines}

Policy information about cell lines

Cell line source(s)

SH-SY5Y from ATCC (ATCC number CRL-2266 ${ }^{\mathrm{TM}}$ ) (lot number 62431864)

SH-SY5Y in house collection

HEK-293T from ATCC (https://www.lgcstandards-atcc.org/Products/All/CRL-11268.aspx?geo_country=be)

Authentication

SH-SY5Y (ATCC); were certified by ATCC by STR genotype analysis (https://www.lgcstandards-atcc.org/en/Products/All/ CRL-2266.aspX\#documentation).

SH-SY5Y cells; (from the in house collection) were authenticated via DNA fingerprinting (Leibniz-Institut DSMZ-Deutsche Sammlung von Mikroorganismen und Zellkulturen $\mathrm{GmbH}$ ).

HEK-293T (ATCC); were certified by ATCC by STR genotype analysis (https://www.lgcstandards-atcc.org/Products/All/ CRL-11268.aspx?geo_country=be\#documentation)

Mycoplasma contamination

All cell lines were routinely tested for mycoplasma contamination using the MycoAlert Mycoplasma Detection kit (LT07-418) and no contaminations have been detected.

Commonly misidentified lines (See ICLAC register)

No commonly misidentified cell lines were used.

\section{Animals and other organisms}

Policy information about studies involving animals; ARRIVE guidelines recommended for reporting animal research

Laboratory animals Mice - E16 FVB/N mice (females for embryos, neuronal isolation). C. elegans - N2 Bristols (Males and females, larvae to adult growth analysis)

Wild animals

No wild animals were used within this research

Field-collected samples

No field collected samples were used within this research

Ethics oversight

All mouse experiments were carried out in accordance with the European Communities Council Directive of November 24, 1986 (86/609/EEC) and approved by the Bioethical Committee of the KU Leuven (Belgium) (ECD project P185-2014).

Note that full information on the approval of the study protocol must also be provided in the manuscript.

\section{Flow Cytometry}

\section{Plots}

Confirm that:

$\square$ The axis labels state the marker and fluorochrome used (e.g. CD4-FITC).

$\square$ The axis scales are clearly visible. Include numbers along axes only for bottom left plot of group (a 'group' is an analysis of identical markers).

$\square$ All plots are contour plots with outliers or pseudocolor plots.

$\triangle \mathrm{A}$ numerical value for number of cells or percentage (with statistics) is provided.

\section{Methodology}

Sample preparation

Instrument

Software

Cell population abundance

Gating strategy

$\triangle$ Tick this box to confirm that a figure exemplifying the gating strategy is provided in the Supplementary Information.

Samples were harvested by TRYPLE, washed and stained. Prior to FACS, cells were resuspended in PBS containing BSA, filtered and stored on ice. Analysis was performed on living (non-fixed) cells.

Attune NxT - Thermo Fisher Scientific

Attune Cytometric Software (v2.1)

Flow cytometry was performed on cell lines and at no point tracked a subpopulation of a heterogeneous cell mixture. We assessed 10,000 events of the total cell population, including both living and dead cells. each cell line. Detection was obtained within the relevant detection window.

Total cells were gated (R1) to remove any signal contamination from debris. Upon selection live/dead cells were characterized in (RX/RY) or fluorescence acquired (RZ). In all cases samples were gated on the position of the negative population (unstained) of 